\title{
The Zona Incerta Regulates Communication between the Superior Colliculus and the Posteromedial Thalamus: Implications for Thalamic Interactions with the Dorsolateral Striatum
}

\author{
Glenn D.R. Watson, ${ }^{1,2}$ Jared B. Smith, ${ }^{2,3}$ and Kevin D. Alloway ${ }^{1,2}$ \\ ${ }^{1}$ Department of Neural and Behavioral Sciences, Pennsylvania State University College of Medicine, Hershey, Pennsylvania $17033-2255$, and ${ }^{2}$ Center for \\ Neural Engineering and ${ }^{3}$ Department of Engineering Science and Mechanics, Pennsylvania State University, University Park, Pennsylvania 16802
}

There is uncertainty concerning the circuit connections by which the superior colliculus interacts with the basal ganglia. To address this issue, anterograde and retrograde tracers were placed, respectively, into the superior colliculus and globus pallidus of Sprague-Dawley rats. In this two-tracer experiment, the projections from the superior colliculus terminated densely in the ventral zona incerta (ZIv), but did not overlap the labeled neurons observed in the subthalamic nucleus. In cases in which anterograde and retrograde tracers were placed, respectively, in sensory-responsive sites in the superior colliculus and posteromedial (POm) thalamus, the labeled projections from superior colliculus innervated the ZIv regions that contained the labeled neurons that project to POm. We also confirmed this colliculo-incertal-POm pathway by depositing a mixture of retrograde and anterograde tracers at focal sites in ZIv to reveal retrogradely labeled neurons in superior colliculus and anterogradely labeled terminals in POm. When combined with retrograde tracer injections in POm, immunohistochemical processing proved that most ZIv projections to POm are GABAergic. Consistent with these findings, direct stimulation of superior colliculus evoked neuronal excitation in ZIv and caused inhibition of spontaneous activity in POm. Collectively, these results indicate that superior colliculus can activate the inhibitory projections from ZIv to the POm. This is significant because it suggests that the superior colliculus could suppress the interactions between POm and the dorsolateral striatum, presumably to halt ongoing behaviors so that more adaptive motor actions are selected in response to unexpected sensory events.

Key words: neuronal tracing; sensorimotor; somatosensory; thalamostriatal; vision

Significance Statement

By demonstrating that the zona incerta regulates communication between the superior colliculus and the posteromedial thalamus, we have uncovered a circuit that partly explains the behavioral changes that occur in response to unexpected sensory stimuli. Furthermore, this circuit could explain why deep brain stimulation of the zona incerta is beneficial to patients who suffer from Parkinson's disease.

\section{Introduction}

We recently reported that the parafascicular (Pf) and posteromedial (POm) nuclei have distinct anatomical and physiological

Received April 25, 2015; revised May 18, 2015; accepted May 22, 2015.

Author contributions: G.D.R.W., J.B.S., and K.D.A. designed research; G.D.R.W., J.B.S., and K.D.A. performed research; G.D.R.W. and K.D.A. analyzed data; G.D.R.W. and K.D.A. wrote the paper.

This work was supported by NIH Grant NS37532 and Pennsylvania State University. We thank Kyle Gheres for his help in developing the immunohistochemical protocol for visualizing GAD 65/67, and the Microscopy Core in The Huck Institutes of the Life Sciences at Penn State University.

The authors declare no competing financial interests.

Correspondence should be addressed to Dr Kevin D. Alloway, Center for Neural Engineering, Millennium Science Complex, W316, Pennsylvania State University, University Park, PA 18602. E-mail: kda1@psu.edu.

DOI:10.1523/JNEUROSCI.1606-15.2015

Copyright $\odot 2015$ the authors $\quad 0270-6474 / 15 / 359463-14 \$ 15.00 / 0$ properties (Alloway et al., 2014). Thalamostriatal projections from Pf innervate both the dorsomedial and dorsolateral striatum, but those from POm innervate only the dorsolateral striatum (DLS). Furthermore, simultaneous recordings in both nuclei indicate that POm adapts slowly, whereas Pf adapts rapidly during repetitive somesthetic stimulation.

Consistent with these distinctions, Pf and POm are activated by different sources. The Pf nucleus is activated by inputs received from the superior colliculus (Grunwerg and Krauthamer, 1992), which coordinates the neural circuits that mediate responses to unexpected stimuli (Sparks, 1986; Comoli et al., 2003; Cohen et al., 2008). This is significant because the collicular projections to Pf could be responsible for executing specific aspects 
of this behavioral function (Grunwerg and Krauthamer, 1990, 1992).

By comparison, $\mathrm{POm}$ receives inputs from the trigeminal nuclei and sensorimotor cortex (Veinante et al., 2000; Alloway et al., 2003). Synchronous inputs from these sources increase POm responsiveness (Groh et al., 2014), which could contribute to the slow adaptation observed in POm. Slowly adapting responses also appear in the DLS (Mowery et al., 2011), which receives most of its thalamic input from POm (Smith et al., 2012) and relies on stimulus-response (S-R) associations to mediate well learned behavioral routines (Graybiel, 2008; Redgrave et al., 2010b; Balleine and O'Doherty, 2010).

These facts and other evidence indicate that thalamostriatal projections from Pf and POm have opposing functions with respect to using sensory information to modulate striatal processing. Whereas POm projections to DLS convey sensory information that mediates $\mathrm{S}-\mathrm{R}$ associations, activation of $\mathrm{Pf}$ causes a pause in striatal processing that could interrupt an ongoing behavior so that a new one is selected (Ding et al., 2010; Thorn and Graybiel, 2010). If this view is correct, then collicular activation of Pf should be accompanied by concomitant inhibition of POm.

The superior colliculus does not project directly to POm, but it innervates the zona incerta (ZI), which contains GABAergic neurons that project to POm (Barthó et al., 2002; Mitrofanis, 2005). Consistent with this, focal lesions in ZI enhance POm responsiveness to somesthetic stimulation, presumably by destroying the inhibitory projections from ZI (Trageser and Keller, 2004; Lavallée et al., 2005). However, no study has demonstrated that activation of the superior colliculus causes excitation of ZI or inhibition of POm. Furthermore, the influence exerted by the superior colliculus on ZI has been questioned by reports asserting that the superior colliculus projects mainly to the subthalamic nucleus (STN), which lies ventral to the ZI (Coizet et al., 2009; Redgrave et al., 2010a).

Determining that the superior colliculus activates ZI and causes inhibition of POm would suggest that these circuit connections have a role in regulating the POm-DLS interactions that mediate well learned behavioral routines. This is clinically significant because it could explain why deep brain stimulation of ZI alleviates some symptoms in Parkinson's disease (PD), as well as essential tremor (Plaha et al., 2008; Sun et al., 2008; Blomstedt et al., 2012; Caire et al., 2013). Therefore, we used anatomical tracing along with neuronal recording and stimulation techniques to determine whether collicular activation of ZI can suppress neuronal activity in $\mathrm{POm}$.

\section{Materials and Methods}

Experiments were performed on adult male Sprague-Dawley rats (Charles River Laboratories) weighing 250-400 g. All methods followed $\mathrm{NIH}$ guidelines and were approved by the Institutional Animal Care and Use Committee at Pennsylvania State University.

Surgery. Anesthesia was induced by an intramuscular injection of ketamine $\mathrm{HCl}(40 \mathrm{mg} / \mathrm{kg})$ and xylazine $(12 \mathrm{mg} / \mathrm{kg})$. Each rat received intramuscular injections of atropine methyl nitrate $(0.5 \mathrm{mg} / \mathrm{kg})$ to limit bronchial secretions, dexamethasone sodium phosphate $(5 \mathrm{mg} / \mathrm{kg})$ to reduce brain swelling, and enrofloxacin $(2.5 \mathrm{mg} / \mathrm{kg})$ to prevent infection. The trachea was intubated through the oral cavity before placing the rat in a stereotaxic instrument and ventilating it with oxygen. Heart rate, respiratory rate, end-tidal carbon dioxide, and blood oxygen were monitored during surgery (Surgivet capnograph, model V90041). Body temperature was regulated by a rectal probe attached to a homeothermic blanket on the dorsal side of the animal while it lay on a water pad set to $37^{\circ} \mathrm{C}$. Ophthalmic ointment was placed on the eyes to prevent corneal desiccation. Bupivacaine was injected at multiple sites in the scalp, the cranium was exposed, and craniotomies were made over the cerebellum for a ground connection and over frontal cortex to acquire an electrocorticographic (ECoG) record.

Additional craniotomies were made for neuronal recordings and tracer injections in multiple brain regions. Relative to bregma, craniotomies were made to access the globus pallidus $(0.8-1.0 \mathrm{~mm}$ caudal, 5.25 mm lateral), caudal POm (3.6-4.2 mm caudal, 2.4-2.8 mm lateral), ZI (3.6-4.4 mm caudal, 2.3-3.3 mm lateral), and superior colliculus (5.9$6.5 \mathrm{~mm}$ caudal, $0.50-2.4 \mathrm{~mm}$ lateral) as indicated by the atlas of Paxinos and Watson (2005).

Extracellular neuronal recordings. Neurons were recorded in POm, ZI, or SC by a tungsten electrode or by a pipette filled with either hypertonic saline or a tracer that could be expelled iontophoretically. A SciWorks data acquisition system (v6.0, DataWave Technologies) was used to display ECoG signals recorded from frontal cortex to indicate the depth of anesthesia. A color-coded fast Fourier transform of ECoG activity was displayed on-line during surgery and subsequent neuronal recordings to visualize changes in cortical frequencies. As ketamine and xylazine were metabolized, the power spectra gradually shifted from $1 \mathrm{~Hz}$ to $5-6 \mathrm{~Hz}$, which indicates a lightly anesthetized state in which the animal is still unconscious (Friedberg et al., 1999). To prevent wakefulness, the rat was continuously ventilated with isoflurane $(0.75-1.25 \%)$ for the rest of the procedure.

Electrodes were advanced vertically through the brain to reach POm (4.9-5.6 mm ventral), ZI (6.8-7.2 mm ventral), or the superior colliculus $(3.2-5.0 \mathrm{~mm}$ ventral). In these regions, neuronal discharges were recorded during whisker deflections or visual stimulation to determine the sensory modality of the recorded neurons. Neuronal discharges were amplified, filtered $(300-3000 \mathrm{~Hz})$, and monitored with an acoustic speaker and a digital oscilloscope. Trial-based responses were displayed on-line and stored on a hard disk. Neuronal responses were also replayed off-line so that discharges could be sorted according to amplitude, width, and other waveform characteristics. Discharges with a signal-to-noise ratio of a least 3:1 were time-stamped at a resolution of $0.1 \mathrm{~ms}$ and were displayed as peristimulus-timed histograms (PSTHs). After measuring the mean rate of spontaneous activity recorded before stimulus onset, confidence limits were displayed on each PSTH, and sensory-evoked responses were considered significant if they exceeded the confidence limits.

Somesthetic and visual stimulation. Whisker deflections were produced by computer-controlled movements of a Galvanometer taken from a Grass Polygraph. As in our previous studies (Zhang and Alloway, 2004; Mowery et al., 2011; Smith et al., 2012), a window screen attached to the tip of the Galvanometer's ink pen was positioned so that multiple whiskers, clipped to a length of 15-20 mm, protruded through the screen by 8-12 mm. A digital waveform generator (ArbStudio by LeCroy) controlled the Galvanometer, and each trial contained a sequence of $50 \mathrm{~ms}$ stimuli in which the whiskers were deflected caudally for $25 \mathrm{~ms}$ and then the Galvanometer returned to the original position in the next $25 \mathrm{~ms}$. In some cases, these pulse-like whisker deflections were followed by sinusoidal whisker movements presented continuously at $8 \mathrm{~Hz}$ for $2 \mathrm{~s}$.

Visual stimulation was administered by a white light source normally used for imaging intrinsic signals from the surface of the neocortex (HAL 100 , Optical Imaging). The light was directed through a pair of opticfiber goosenecks positioned $3-5 \mathrm{~cm}$ away from each eye. On each computer-controlled trial, a single $50 \mathrm{~ms}$ light flash was administered.

Tracer injections. To detect retrogradely labeled connections, we injected $2 \%$ solutions of FluoroGold (FG; H-22845, Fluoro-Chrome) mixed in $0.9 \%$ saline. To detect anterogradely labeled connections, we used several tracers, including a $10 \%$ solution of biotinylated dextran amine (BDA; D-7135, Invitrogen) in $0.01 \mathrm{M}$ PBS, a $10 \%$ solution of Fluoro-Ruby (FR; D-1817, Invitrogen) in 0.01 м PBS, or a $10 \%$ solution of AlexaFluor (AF; D-22910, Invitrogen) in $0.01 \mathrm{M}$ PBS. Both FR and AF were pressure ejected from $2 \mu \mathrm{l}$ Hamilton microsyringes in volumes ranging from 150 to $400 \mathrm{nl}$. Solutions of FG and BDA were iontophoretically expelled from the micropipette by using positive current pulses of 2-5 $\mu \mathrm{A}$ applied for $15-25 \mathrm{~min}$ at each site. For some experiments, a mixture of FG and BDA was iontophoretically ejected from a single pipette to reveal the afferent and efferent connectivity of physiologically 
defined sites in ZI. Retention currents $(-10 \mu \mathrm{A})$ were always applied to FG and BDA solutions to prevent unwanted tracer leakage along the trajectory of the pipette.

The retrograde tracer FG was injected into the external globus pallidus (GP) at depths of 5.5-5.8 $\mathrm{mm}$ (using a $25^{\circ}$ angular approach within the coronal plane) or into the POm at depths of 4.9-5.6 mm. Anterograde tracers were injected either into visually responsive parts of the medial superior colliculus $(0.5-1.0 \mathrm{~mm}$ lateral and $6.1-6.4 \mathrm{~mm}$ caudal to bregma) or into whisker-sensitive parts of the lateral superior colliculus (2.0-2.4 mm lateral and 6.1-6.4 $\mathrm{mm}$ caudal to bregma). The visual and somesthetic sites in superior colliculus were located, respectively, 3.2-3.8 $\mathrm{mm}$ and $3.5-5.0 \mathrm{~mm}$ ventral to the pial surface. Tracers were injected into ZI at sites located $2.5-3.0 \mathrm{~mm}$ lateral and $3.5-4.0 \mathrm{~mm}$ caudal to bregma, and $6.8-7.2 \mathrm{~mm}$ ventral to the pial surface.

Following tracer injections, the skin was sutured and treated with antibiotic ointment. Each animal received additional doses of atropine, dexamethasone, and enrofloxacin. Animals were returned to single-housed cages for a 7-10 d survival period to allow for tracer transport.

Superior colliculus stimulation. A small craniotomy was made over the superior colliculus and a micropipette syringe fitted with a glass pipette (Hamilton) was lowered through the craniotomy to inject 800-1200 $\mathrm{nl}$ of AAV1.CamKIIa.hChR2(H134R)-eYFP.WPRE.hGH(Addgene26969P, Penn Vector Core) at a rate of $60 \mathrm{nl} / \mathrm{min}$. Approximately 10 weeks after injecting the adeno-associated virus (AAV) to induce ChR2 expression, the rat was used in a lightly anesthetized preparation (see Surgery). An optrode consisting of an optic fiber ( $400 \mu \mathrm{m}$ core, $0.39 \mathrm{NA}$, Thor Labs) attached to a tungsten electrode was inserted through the craniotomy and was slowly advanced toward the superior colliculus. The tungsten electrode extended $300 \mu \mathrm{m}$ beyond the tip of the optic fiber, which was connected to a patch cable that transmitted blue light from a laser ( $100 \mathrm{~mW}, 473 \mathrm{~nm}$, Opto Engine). As the optrode was advanced, $50 \mathrm{~ms}$ blue light pulses were administered at $2 \mathrm{~Hz}$ to evoke extracellular discharges from the ChR2-infected neurons in the superior colliculus.

When light-evoked discharges were encountered in the superior colliculus, a second electrode consisting of a saline-filled pipette was advanced through a second craniotomy above ZI. The electrode was slowly advanced toward ZI while $50 \mathrm{~ms}$ pulses of blue light were flashed in the superior colliculus. Upon encountering optically evoked responses in ZI, the electrode position was adjusted to improve the isolation of the extracellular discharges.

For electrical stimulation experiments, a concentric bipolar electrode (FHC) was placed in the superior colliculus while a recording electrode was lowered into POm. To maintain the rat in a stable, lightly anesthetized plane $(0.75 \%$ isoflurane), gooseneck manipulators (Flexbar) were clamped to a pair of large screws embedded in dental cement that covered small machine screws fixed into the cranium at the occipital ridge. The stereotaxic ear bars were removed after the head was held stationary by the gooseneck manipulators. Removing nociceptive activation of the external auditory meatus facilitated spontaneous firing in POm and allowed detection of inhibition. After isolated neurons were encountered in POm, the superior colliculus was electrically stimulated with five $1 \mathrm{~ms}$ duration biphasic pulses (ranging from 200-500 $\mu \mathrm{A}$ ) administered over a $10 \mathrm{~ms}$ period (i.e., at $500 \mathrm{~Hz}$ ).

Histology. Each rat was deeply anesthetized with intramuscular injections of ketamine $(80 \mathrm{mg} / \mathrm{kg})$ and xylazine $(6 \mathrm{mg} / \mathrm{kg})$, and then was perfused transcardially with heparinized saline, $4 \%$ paraformaldehyde, and $4 \%$ paraformaldehyde in $10 \%$ sucrose. The brain was removed and stored in $4 \%$ paraformaldehyde with $30 \%$ sucrose in a refrigerator for $3-5 \mathrm{~d}$. Before sectioning, the cerebellum and olfactory bulbs were removed. In some rats, only one hemisphere was sectioned; in others, both hemispheres were sectioned simultaneously after a narrow slit was made in the left hemisphere to indicate the correct left-right orientation. A freezing microtome was used to cut each brain into serially ordered 60$\mu \mathrm{m}$-thick sections that were placed in $0.1 \mathrm{M}$ PBS.

In cases in which animals were used for neuronal tracing, a 1-in-2 or 1-in-3 series of sections were processed and mounted onto gel-dipped slides in serial order. The first series, used to visualize cytoarchitecture, was either stained with thionin or processed for the presence of cyto- chrome oxidase (Land and Simons, 1985). A second series, used for visualizing fluorescent tracers (i.e., FR, FG, AF), did not undergo any histochemical processing. In most cases, a third series was used to visualize BDA labeling (Kincaid and Wilson, 1996; Smith et al., 2012). The BDA-labeled sections were briefly agitated in $0.3 \% \mathrm{H}_{2} \mathrm{O}_{2}$ and then placed in $0.1 \mathrm{M}$ PBS with $0.3 \%$ Triton X-100, pH 7.4, before incubating them for $2-4 \mathrm{~h}$ in an avidin-biotin horseradish peroxidase solution (Vector Novocastra Laboratories). After rinsing sections in PBS, the tracer was visualized with $0.05 \%$ diaminobenzidine (DAB), $0.005 \% \mathrm{H}_{2} \mathrm{O}_{2}, 0.04 \%$ $\mathrm{NiCl}_{2}$, and $0.04 \% \mathrm{CoCl}_{2}$ in $0.1 \mathrm{M}$ Tris buffer, $\mathrm{pH} 7.2$, for $9-12 \mathrm{~min}$. The $\mathrm{DAB}$ reaction was stopped by subsequent washes in PBS. Mounted sections in each series were dehydrated in ethanol, defatted in xylene, and coverslipped with cytoseal.

GAD processing. To visualize neurons in $\mathrm{ZI}$ that contained GABA, coronal sections were processed for glutamic acid decarboxylase (GAD) using procedures adapted from previous reports (Oostland et al., 2011; Aligny et al., 2014). The sections were rinsed in $0.1 \mathrm{M}$ PBS for $20 \mathrm{~min}$ before being placed in a PBS-based blocking solution that contained 0.3 $\mathrm{M}$ glycine, $5 \%$ goat serum, $1 \%$ bovine serum albumin, and $0.1 \%$ Triton $\mathrm{X}-100, \mathrm{pH} 7.4$, at room temperature for $1 \mathrm{~h}$. Sections were then incubated overnight with polyclonal rabbit anti-GAD 65/67 antibody (1:250, ab49832, Abcam) diluted in PBS at $4^{\circ} \mathrm{C}$. Sections were rinsed in PBS for 20 min before being placed in DyLight 488 anti-rabbit IgG (1:250, Vector Laboratories) secondary antibody diluted in blocking buffer for $1 \mathrm{~h}$ at room temperature. Sections were then rinsed with PBS, defatted with xylene, and coverslipped. High-resolution fluorescent images were acquired using a Fluoview 1000 laser-scanning confocal imaging system (Olympus Optical).

Anatomical analysis. We used an Olympus BH-2 microscope equipped for fluorescent microscopy to reconstruct anterograde and retrograde labeling. An Accustage plotting system was used to plot labeled cell bodies and axonal varicosities relative to well known anatomical landmarks. The BDA-labeled terminals were viewed in bright-field illumination, and fluorescent-labeled soma and terminals were visualized using a TRITC filter (41002; Chroma Technologies). Axonal varicosities were plotted because these represent en passant synapses (Voigt et al., 1993; Kincaid and Wilson, 1996; Meng et al., 2004). Anatomical landmarks and tracer labeling were also photographed by a Retiga EX CCD digital camera (Q-imaging) mounted on the microscope.

A module in the Accustage software was used to quantify terminalsoma overlap in ZI for those cases in which a retrograde tracer deposit in POm was paired with a tracer injection in the superior colliculus. Plotted reconstructions were subdivided into $50 \mu \mathrm{m}^{2}$ bins as in our previous reports (Smith and Alloway, 2010, 2013). Each bin with at least one FG-labeled soma was colored gold, those with two or more FR-labeled varicosities were colored red, and those with an FG-labeled cell and at least two FR-labeled varicosities were colored white.

\section{Results}

Data were obtained from a total of 35 rats. In three rats, the tracers FG and BDA were injected into the globus pallidus and $\mathrm{SC}$, respectively, to visualize the specificity of collicular projections to STN or ZI. In 18 rats, an injection of FG in POm was paired with one or two anterograde tracer injections in superior colliculus to determine whether the superior colliculus innervates $\mathrm{ZI}$ regions that project to $\mathrm{POm}$. In seven rats, physiologically defined parts of ZI were injected with a combined solution of FG and BDA to confirm the input-output connectivity of ZI. In the remaining seven rats, we activated the superior colliculus, either optogenetically or with conventional electrical stimulation, while recording neuronal responses in ZI or POm.

\section{Nonoverlapping connections of the superior colliculus and globus pallidus}

In three rats, we injected anterograde and retrograde tracers, respectively, into the superior colliculus and lateral GP. The STN has both afferent and efferent connections with the lateral GP 

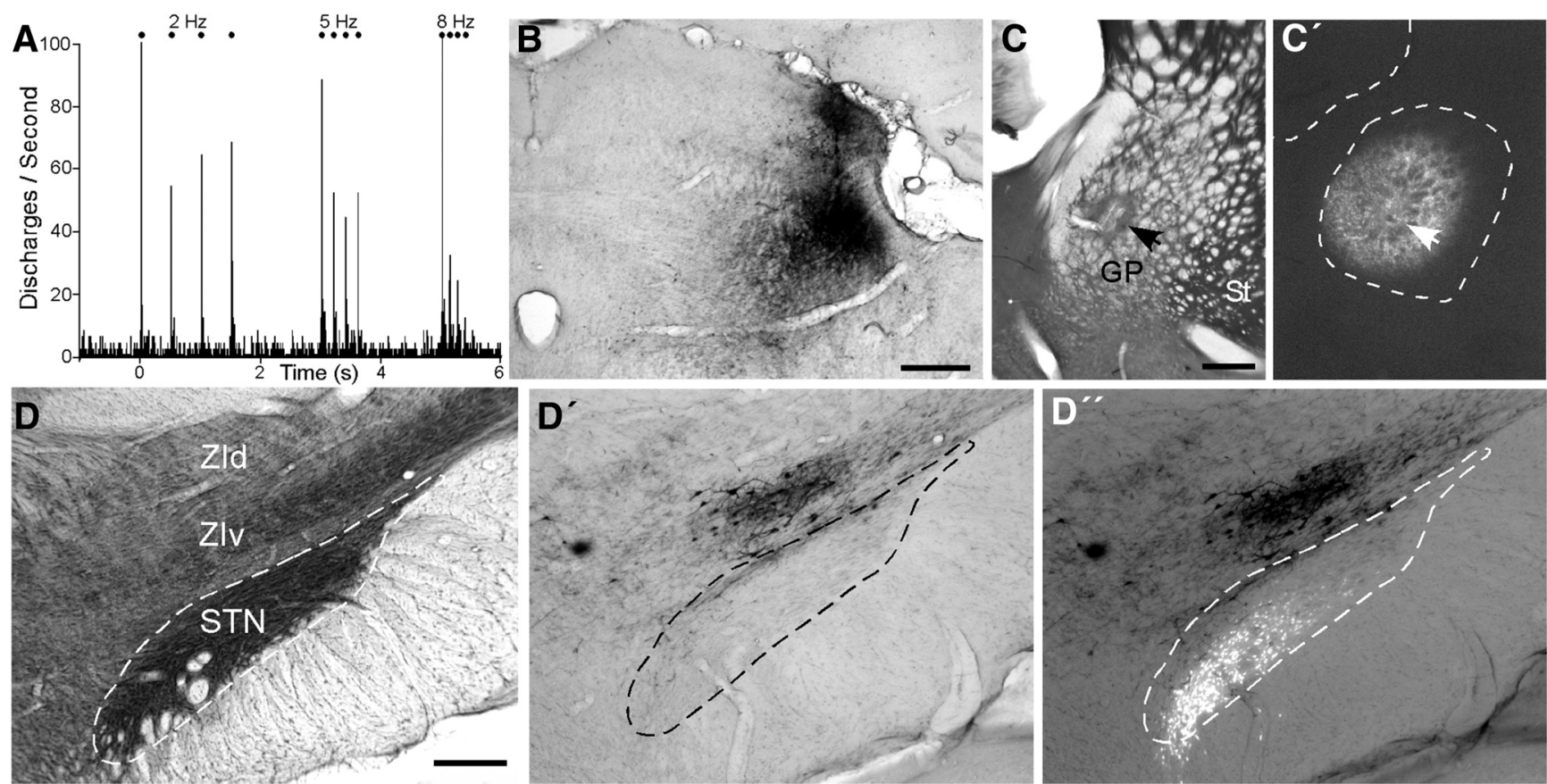

Figure 1. Superior colliculus projects predominantly to Zlv, not to the STN. A, PSTH showing multiunit responses to whisker deflections recorded by a BDA-filled pipette in the lateral part of the superior colliculus. Binwidths, $10 \mathrm{~ms}$. B, Deposit of BDA at the site of the neural responses shown in $A$. C, Section through the globus pallidus (GP) and striatum (St) processed for C0. Note the necrosis at the tracer injection site (arrow) in GP. $C^{\prime}$, Adjacent section through GP shows the widest extent of the FG deposit in GP. $\boldsymbol{D}, C 0$-processed section through Zl and STN. $\boldsymbol{D}^{\prime}, \boldsymbol{D}^{\prime \prime}$, Adjacent section viewed with conventional and fluorescent illumination indicates that BDA-labeled terminals and cell bodies in Zlv are separate from the FG-labeled cells in STN. Scale bars: $\boldsymbol{B}, \boldsymbol{C}, \boldsymbol{C}^{\prime}, 500 \mu \mathrm{m} ; \mathbf{D}, \boldsymbol{D}^{\prime}, \boldsymbol{D}^{\prime \prime}$, $250 \mu \mathrm{m}$.

(Kita and Kitai, 1987; Groenwegen and Berendse, 1990), and we conducted these paired tracer experiments to determine whether projections from the superior colliculus terminate in the STN as defined by its connectivity with the GP.

As shown in Figure 1, we used a BDA-filled pipette to record whisker-responsive neurons in the intermediate and deep layers of the lateral superior colliculus. After recording responses to repetitive whisker deflections, the tracer was iontophoretically injected at the recording site (Fig. $1 A, B$ ). This is the same region that was injected with anterograde tracers in previous studies that reported collicular projections to STN (Tokuno et al., 1994; Coizet et al., 2009; Redgrave et al., 2010a).

Subsequently, FG was placed in the lateral GP to reveal retrogradely labeled neurons in STN. Using stereotaxic coordinates (Paxinos and Watson, 2005), FG was iontophoretically ejected into the center of the lateral GP (see Fig. $1 C$ ). Although the tracer diffused across a wide expanse of the lateral GP, it did not enter the striatum or surrounding regions (Fig. $1 C^{\prime}$ ).

As indicated by Figure $1 D$, the STN is easily recognized in coronal sections processed for cytochrome oxidase (CO). In contrast to ZI, which contains moderate concentrations of $\mathrm{CO}$ in its ventral tier, STN is noticeably darker and is located immediately above the cerebral peduncle, which is completely devoid of CO.

In alternate sections processed for BDA, the labeled projections from superior colliculus terminated mainly in ZIv, which lies above STN (Fig. 1D'). A few BDA-labeled terminals entered STN, but these were diffusely scattered along its dorsal edge and did not form any dense patches. Although BDA is transported primarily in the anterograde direction, some BDA-labeled neurons were observed in ZIv, which is consistent with known projections from ZI to the superior colliculus (Mitrofanis, 2005). In no instance, however, did we ever observe any retrogradely labeled neurons in STN after placing BDA in the superior colliculus.
In sections processed for BDA, we were able to use fluorescent microscopy to visualize the FG-labeled neurons in STN. In all cases, dense aggregates of FG-labeled neurons appeared in the STN region marked by high concentrations of $\mathrm{CO}$ in adjacent sections (Fig. 1, compare $D, D^{\prime \prime}$ ). The BDA-labeled terminals and neurons were also apparent during fluorescent illumination, but consistent with their location within the ventral ZI, the regions labeled by FG and BDA were completely separate.

\section{Overlapping connections in the zona incerta}

To determine whether the superior colliculus innervates ZI regions that project to $\mathrm{POm}$, anterograde and retrograde tracers were injected into the superior colliculus and POm, respectively. In all cases, the retrograde tracer was placed in caudal POm, which project to DLS (Ohno et al., 2012; Alloway et al., 2014). Figure $2\left(A, B, B^{\prime}\right)$ depicts the location of a FG deposit at a POm site that responded to whisker stimulation but was unresponsive to visual stimulation. In the same rat, BDA was placed at a site in medial superior colliculus that consistently responded to visual stimulation but not to whisker deflections (Fig. 2C, D, $D^{\prime}$ ).

The BDA-labeled collicular projections terminated in the lateral part of ZIv without encroaching upon the dorsal border of STN (Fig. 2E-G). This crossed topographic pattern, in which the medial superior colliculus projects to lateral ZI, was observed in each of the 12 cases in which an anterograde tracer was placed medially in collicular regions that responded primarily to light stimulation.

Consistent with previous work (Lavallée et al., 2005; Alloway et al., 2014), many ZI neurons were retrogradely labeled after we injected FG into whisker-sensitive parts of POm. Furthermore, this ZIv region also received BDA-labeled projections from the light-sensitive region in the superior colliculus (Fig. 2G). This is a significant finding because it demonstrates a cross-modal circuit 

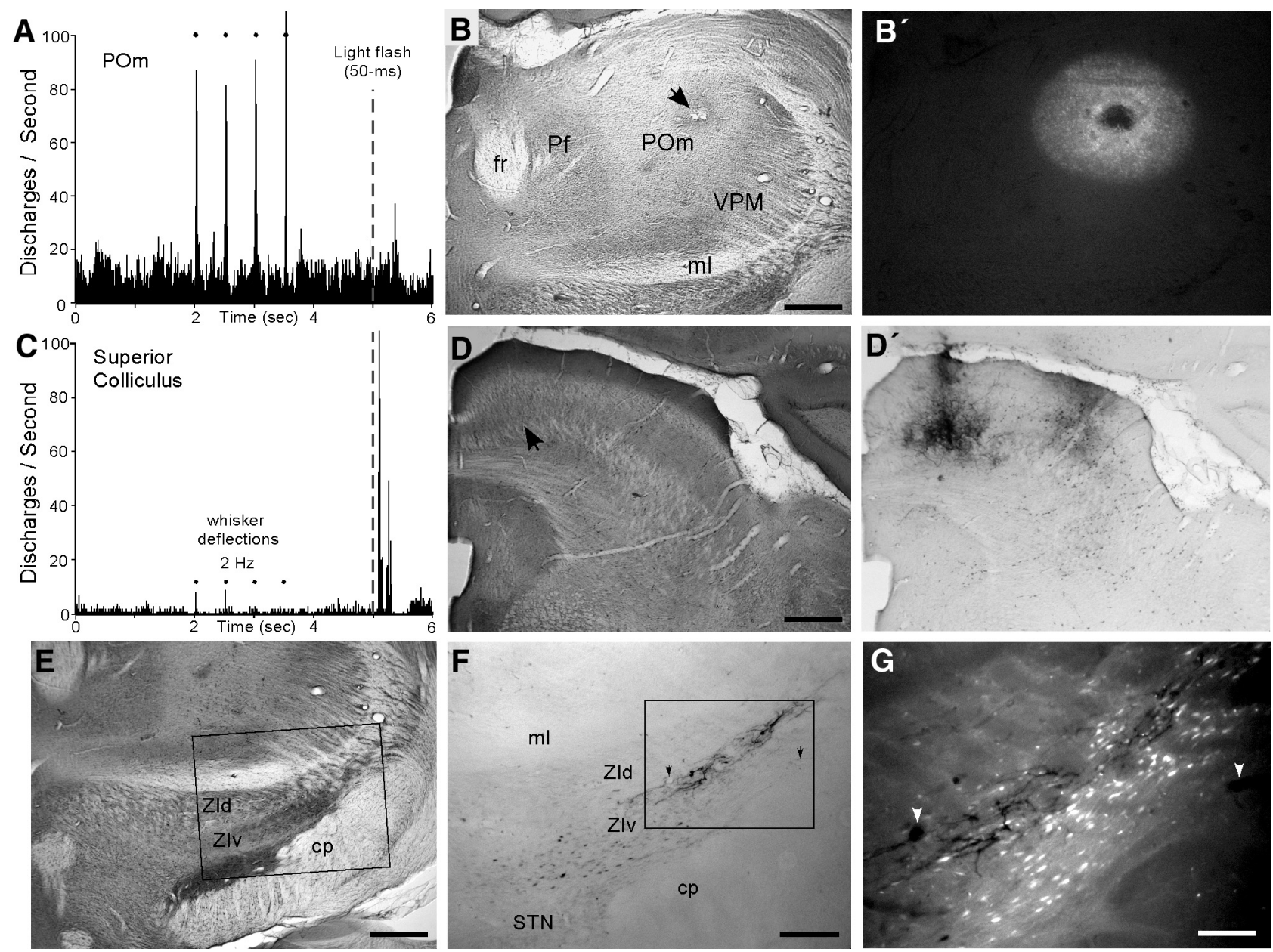

Figure 2. Light-responsive region in superior colliculus projects to Zlv region that innervates whisker-responsive region in POm. $A$, PSTH shows whisker-induced multiunit responses recorded in POm by an FG-filled pipette. This site did not respond to a $50 \mathrm{~ms}$ light flashed in both eyes. $\boldsymbol{B}$, Section through POm indicates necrosis at the FG deposit marking the whisker-responsive site in $\boldsymbol{A}$. $\boldsymbol{B}^{\prime}$, Same section under fluorescent illumination shows extent of the FG deposit. C, Multiunit responses to visual light flash recorded in the medial superior colliculus by a BDA-filled pipette. Neurons at this site responded weakly to whisker stimulation. $\boldsymbol{D}$, C 0 -processed section of the recording site (arrow) in the medial superior colliculus. $\boldsymbol{D}^{\prime}$, Adjacent section shows the BDA deposit marking the light-responsive site in C.E, CO-processed section through ZI and STN. Inset indicates region displayed in F.F, Adjacent section shows BDA-filled cells and terminals in upper part of Zlv. Inset indicates the region displayed in $\boldsymbol{G}$. G, Fluorescent illumination at high-magnification reveals BDA-labeled terminals intermingled with FG-labeled neurons in Zlv. Arrows indicate same blood vessels in $\boldsymbol{F}$ and G. Scale bars: $\boldsymbol{B}, \boldsymbol{B}^{\prime}, \boldsymbol{D}, \boldsymbol{D}^{\prime}, \boldsymbol{E}, 500 \mu \mathrm{m} ; \boldsymbol{F}, 250 \mu \mathrm{m} ; \boldsymbol{G}, 100 \mu \mathrm{m}$.

in which light-responsive sites in superior colliculus project to ZIv regions that innervate whisker-sensitive sites in POm.

\section{Quantitative analysis of tracer overlap in the zona incerta}

In 7 of 18 rats that received tracer deposits in both the superior colliculus and POm, the tracers were injected at sites that responded primarily to whisker stimulation. Figure $3\left(A, B, B^{\prime}, B^{\prime \prime}\right)$ illustrates one case in which FG was placed in POm and FR was injected in the lateral superior colliculus $\left(C, D, D^{\prime}, E\right)$. Confocal images obtained from this case indicated that several sites in ZIv were occupied by FR-labeled terminals that overlapped the FG-labeled soma (Fig. $\left.3 G, G^{\prime}, G^{\prime \prime}\right)$.

The locations of labeled cells and terminals were plotted and reconstructed as shown in Figure $4(A-C)$. Each reconstructed section was subdivided into a grid of $50 \mu \mathrm{m}^{2}$ bins, and each bin was color-coded by whether it contained labeled neurons (gold), labeled terminals (red), or both labeled neurons and terminals (white). Figure $4\left(A^{\prime}-C^{\prime}\right)$ indicates that the proportion of bins that contained both tracers varied across sections, but appeared to be greatest in ZIv.

To quantify the regional distribution of labeling, all plotted cells and terminals in the subthalamic region were counted and classified according to their location in STN or in the dorsal, ventral, rostral, and caudal sectors of ZI. The regional subdivisions of ZI were defined by the presence of $\mathrm{CO}$ and other anatomical landmarks as depicted in other publications (Paxinos and Watson, 2005; Watson et al., 2014). The number of $50 \mu \mathrm{m}^{2}$ bins that contained both labeled cells and terminals were counted and classified by their location in the different sectors of ZI. Because the amount of labeling varied across individual cases according to the spatial extent of the tracer injections, for each rat we normalized the data by expressing the labeling in each region as a proportion of the total amount of labeling across all subthalamic regions.

The regional distributions of labeled terminals, labeled cells, and labeled overlap are presented in Figure 5. On average, the STN received $\sim 2 \%$ of the labeled projections from the superior colliculus, but did not contain any labeled cells that project to POm (Fig. 5A, B). By contrast, ZIv contained nearly one-half of the plotted terminals and slightly more than one-half of the plotted cells. Consequently, most tracer overlap, in which two labeled terminals were in the same bin as a labeled neuron (i.e., terminal-somal overlap), was observed in ZIv. An ANOVA indicated a significant regional effect for each distribution depicted in Figure 5 ( $F \geq 20.8, p<0.0001$ for all anal- 

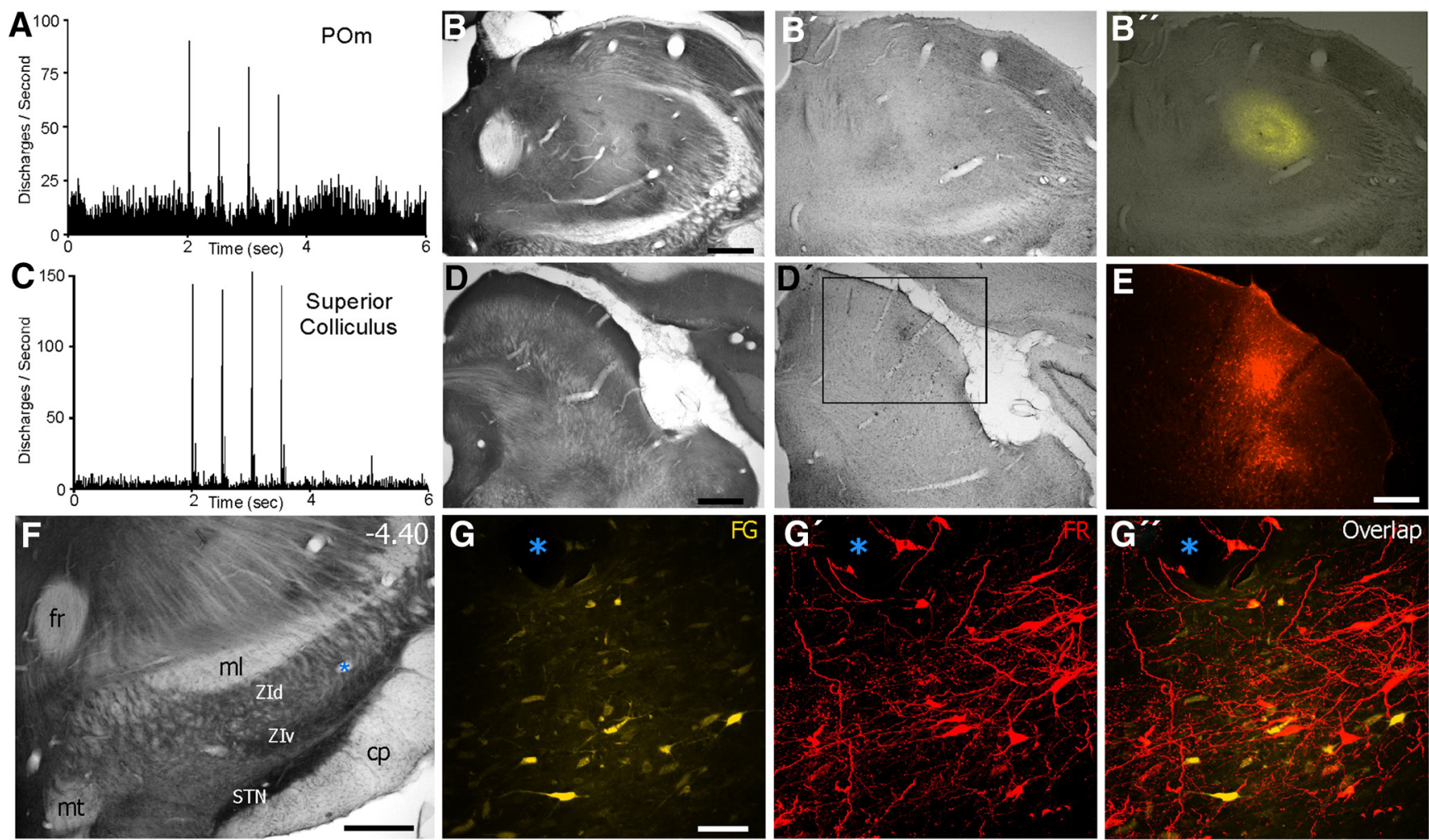

Figure 3. Projections from the superior colliculus terminate in Zlv regions that project to $\mathrm{PO}$. $A$, Whisker deflections at $2 \mathrm{~Hz}$ evoke POm multiunit responses recorded by a FG-filled pipette. $\boldsymbol{B}$, Section processed for $\mathrm{CO}$ shows $\mathrm{POm} . \boldsymbol{B}^{\prime}, \boldsymbol{B}^{\prime \prime}$, Adjacent section viewed conventionally and with fluorescent microscopy depicts the $\mathrm{FG}$ deposit in POm. $\boldsymbol{C}$, Multiunit response to whisker deflections recorded in superior colliculus of the same rat. $\boldsymbol{D}, \boldsymbol{D}^{\prime}, \boldsymbol{E}$, Adjacent sections processed $(\boldsymbol{D})$ and unprocessed $\left(\boldsymbol{D}^{\prime}, \boldsymbol{E}\right)$ for $\mathbf{C} 0$ depict the neuronal recording site and FR deposit in the intermediate layers of the superior colliculus. $\boldsymbol{D}^{\prime}$, Inset indicates the region shown in $\boldsymbol{E} . \boldsymbol{F}, \mathbf{C} 0$-processed section through Zl and STN. Inset indicates the region selected for confocal microscopy in subsequent panels. $\boldsymbol{G}$, $\boldsymbol{G}^{\prime}, \boldsymbol{G}^{\prime \prime}$, Confocal images illustrate overlap of FG-labeled neurons and FR-labeled terminals and neurons in Zlv. Blue asterisk indicates the same blood vessel in $\boldsymbol{F}$. Scale bars: $\boldsymbol{B}, \boldsymbol{D}, \boldsymbol{F}, 500 \mu \mathrm{m} ; \boldsymbol{E}, 250$ $\mu \mathrm{m} ; \mathbf{G}, 50 \mu \mathrm{m}$. cp, Cerebral peduncle; fr, fasciculus retroflexus; ml, medial lemniscus; $\mathrm{mt}$, mammilothalamic tract.
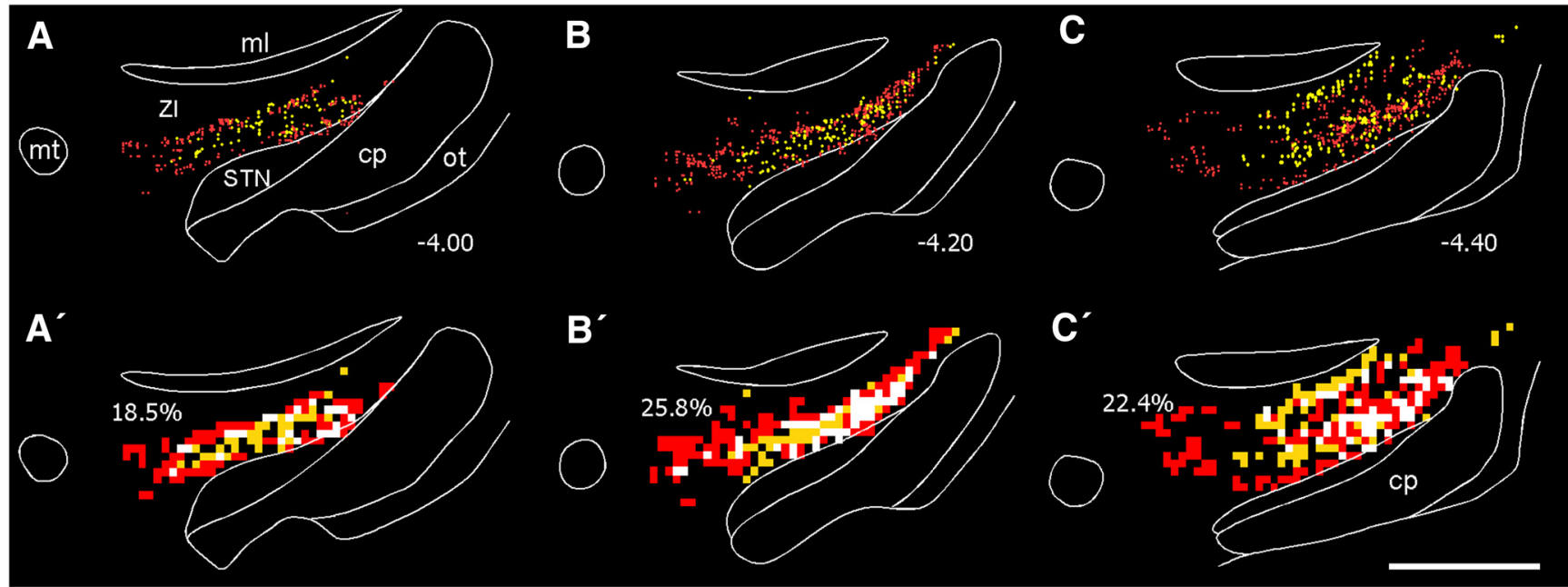

Figure 4. Collicular projections overlap the ZI neurons that project to POm. A-C, Reconstructed plots of FG-filled neurons (gold) and FR-labeled terminal varicosities (red) in Zl. Numbers indicate distance from bregma in millimeters. $A^{\prime}-C^{\prime}, 0$ verlap analysis of FG-labeled neurons and FR-labeled terminals in Zl, using $50 \mu \mathrm{m}^{2}$ bins. Each panel corresponds to the plotted reconstruction shown directly above. For the overlap analysis, gold bins contain at least one FG-labeled neuron, red bins contain at least two FR-labeled terminals, and white bins contain at least one FG-labeled neuron and two FR-labeled terminals. Percentages indicate proportion of white bins. Scale bar, $1 \mathrm{~mm}$. cp, Cerebral peduncle; $\mathrm{ml}$, medial lemniscus; mt, mammilothalamic tract; ot, optic tract.

yses). The amount of tracer overlap was significantly greater in ZIv than in any other sector (paired $t$ tests $\geq 4.5, p<0.01$ for all comparisons), but there were no differences between ZIr, ZId, and ZIc. Input-output connectivity of ZIv

To characterize the input-output connectivity of ZIv more completely, we iontophoretically deposited a mixture of FG and BDA into physio- logically defined sites in ZI of seven rats. Deposits of FG/BDA occupied multiple zones of ZI or else diffused into surrounding nuclei in four of the rats, but in the remaining three rats the tracers were confined to ZIv. Combining FG with BDA increases the retrograde transport of BDA and permits the use of conventional light microscopy at lowmagnifications so that we could photograph populations of labeled neu- 


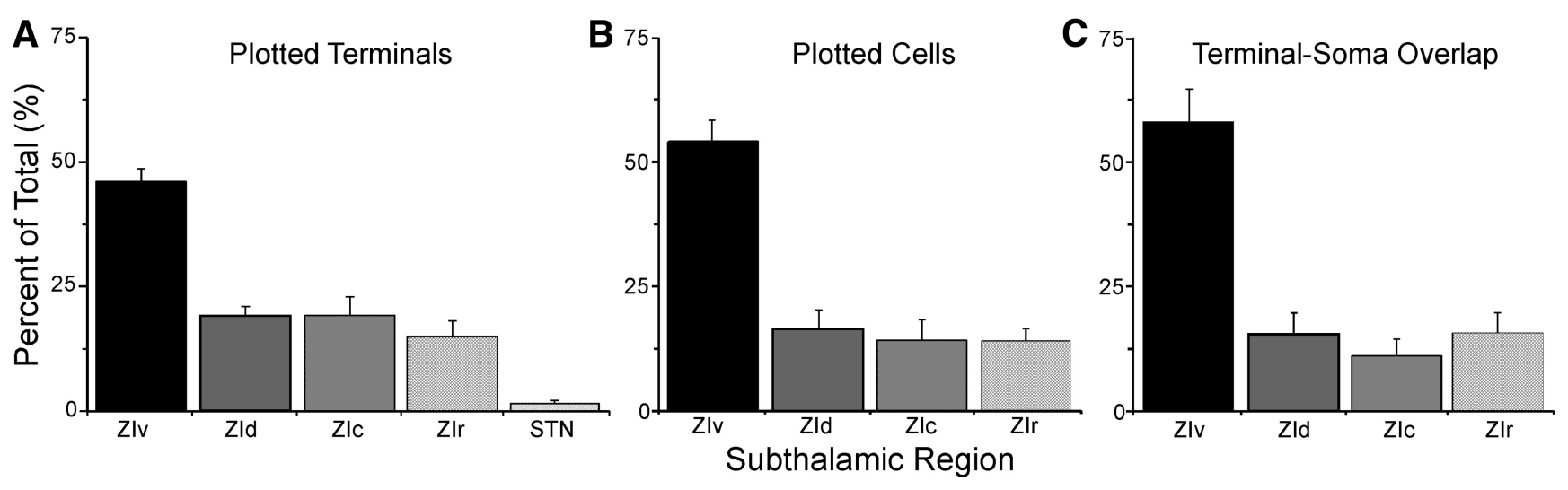

Figure 5. Normalized distributions of labeling across the subthalamic subdivisions. $\boldsymbol{A}$, Distribution of plotted terminals produced by anterograde tracer injections in whisker-responsive sites of the superior colliculus. $\boldsymbol{B}$, Distribution of plotted cells produced by retrograde tracer injections in whisker responsive-sites in POm. C, Distribution of terminal-soma overlap based on the proportion of $50 \mu \mathrm{m}^{2}$ bins that contained at least one labeled cell and two labeled terminals.
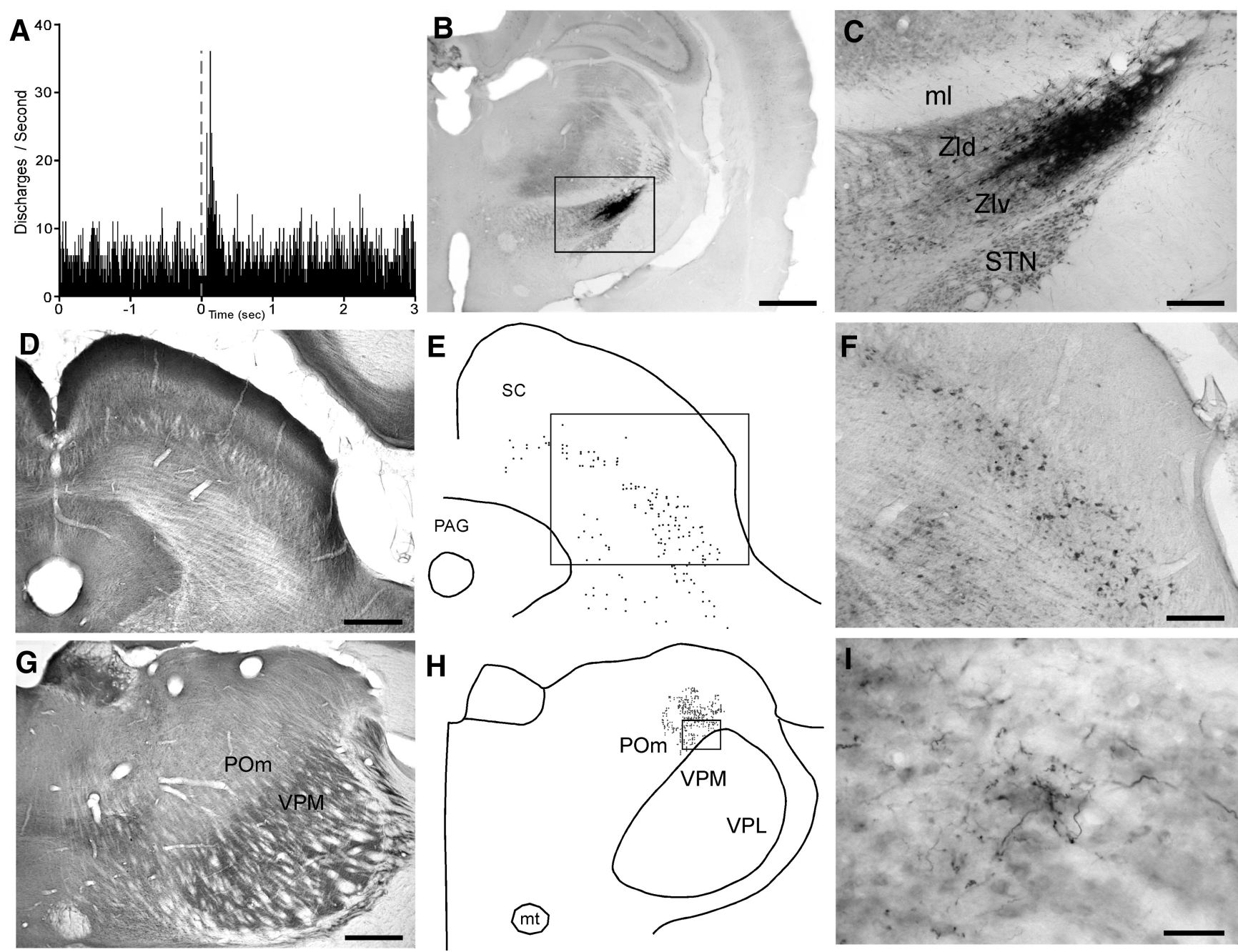

Figure 6. Afferent and efferent connectivity of a visually responsive region in ZIv. A, PSTH shows neural responses in lateral Zlv to a $50 \mathrm{~ms}$ visual light flash recorded by a pipette filled with both FG and BDA. B, C, BDA deposit at the light-sensitive site recorded in $A$. D, C0-processed section through the superior colliculus. $E$, $F$, Plotted reconstruction and photomicrograph of BDA-labeled neurons in superior colliculus; rectangle indicates region in $\boldsymbol{F} . \mathbf{G}, \mathbf{C} 0$-processed section through $\mathrm{PO}$ m and VPM. $\boldsymbol{H}, \boldsymbol{I}$, Plotted reconstruction and photomicrograph of BDA-labeled terminal varicosities in POm; rectangle indicates the region in $I$. Scale bars: $B, D, G, 500 \mu \mathrm{m} ; \boldsymbol{F}, 250 \mu \mathrm{m} ; \boldsymbol{C}, 100 \mu \mathrm{m} ; \boldsymbol{I}, 50 \mu \mathrm{m}$. PAG, Periaqueductal gray; VPM, ventroposteromedial; VPL, ventroposterolateral.

rons distributed across broad regions of tissue (Smith et al., 2012; Alloway et al., 2014).

Figure 6 illustrates a case in which FG and BDA were simultaneously ejected at a site in lateral ZIv that responded to visual stimulation. The tracers occupied an oval-shaped region that extended $\sim 400 \mu \mathrm{m}$ along the mediolateral axis of ZI, but they did not diffuse into STN or other nearby structures. 

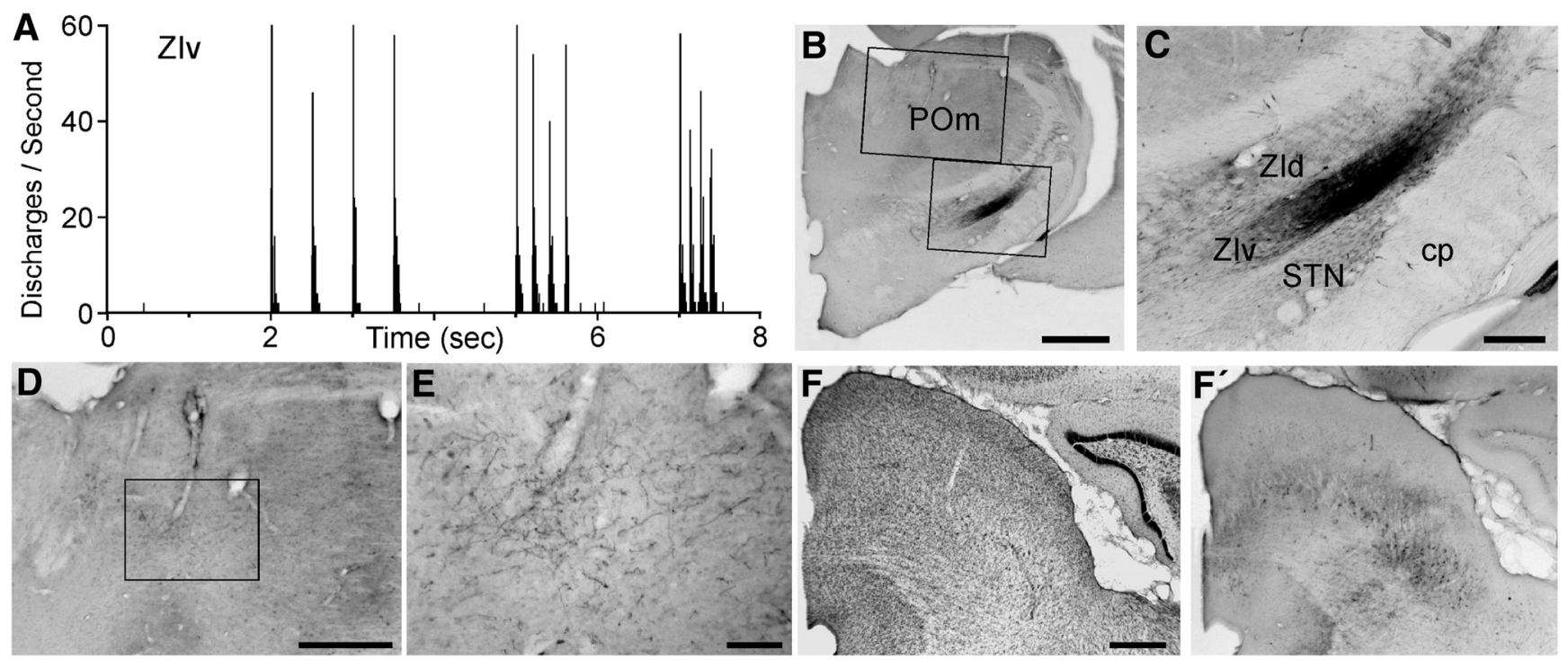

Figure 7. Afferent and efferent connections of a whisker-responsive region in Zlv. A, PSTH shows neural responses in Zlv recorded by a FG/BDA-filled pipette during whisker deflections administered at 2,5, and $8 \mathrm{~Hz} . \boldsymbol{B}, \boldsymbol{C}, \mathbf{B D A}$ deposit at the site of the whisker-responses recorded in $\boldsymbol{A}$. Rectangles in $\boldsymbol{B}$ indicate regions in $\mathbf{C}$ and $\boldsymbol{D}$. D, E, Low-and high-power views of BDA-labeled axonal terminals in POm. $\boldsymbol{D}$, Inset indicates region in $\boldsymbol{E}$. $\boldsymbol{F}$, Thionin-stained section through the superior colliculus. $\boldsymbol{F}^{\prime}$, Adjacent section showing the laminar location and spatial extent of BDA-labeled neurons in the superior colliculus. Scale bars: $\boldsymbol{B}, 1 \mathrm{~mm} ; \boldsymbol{D}, \boldsymbol{F}, 500 \mu \mathrm{m} ; \boldsymbol{C}, 250 \mu \mathrm{m} ; \boldsymbol{E}, 100 \mu \mathrm{m}$.

Consistent with other reports of a colliculo-incertal pathway (Roger and Cadusseau, 1985; Shammah-Lagnado et al., 1985), the combined injection of FG and BDA revealed a large number of labeled neurons in the intermediate and deep layers of the superior colliculus (Fig. 6D-F). Surprisingly, neuronal labeling was continuously distributed across a broad expanse of superior colliculus that extended from sites above the periaqueductal gray to the most lateral part of the superior colliculus. Based on our recordings throughout the superior colliculus of other experiments, this neuronal labeling appeared to occupy both somesthetic and visually responsive regions. Neurons recorded at the ZI injection site responded specifically to light stimulation (manual whisker deflections did not evoke any response in this case), but some of the injected tracer diffused medially within ZI and may account for the retrograde labeling in the lateral superior colliculus.

Deposits of anterograde tracers in ZIv were always accompanied by terminal labeling in POm. Figure 6 demonstrates that the combined BDA/FG tracer deposit in lateral ZIv produced a dense concentration of BDA-labeled terminals and varicosities in the lateral POm region that adjoined VPM. There was no terminal labeling in VPM, however. In fact, as indicated by our photomicrographs and plotted reconstructions of labeled varicosities (Fig. 6G-I), the boundary separating POm and VPM was practically defined by labeled terminals concentrated exclusively on the POm side of this border. The location of these labeled terminals in $\mathrm{POm}$ is consistent with whisker-sensitive POm sites in other studies (Diamond et al., 1992, Alloway et al., 2003; Smith et al., 2012), and this indicates that the light-sensitive region in ZIv projects to whisker-responsive regions in POm.

Figure 7 illustrates the distribution of labeling in superior colliculus and POm from another case in which a combination of FG and BDA was placed in ZIv. In contrast to the case presented in Figure 6, however, this combined tracer injection was placed more medially at a ZI site that responded to whisker deflections but not to visual stimulation. Despite this difference in the functional location of the tracer deposit, the labeling patterns in the superior colliculus and POm resembled the input-output patterns depicted in Figure 6.

In all three cases in which the FG/BDA deposit was confined to ZIv, the labeled projections terminated throughout the POm, including both its rostral and caudal portions. This could be significant because both rostral and caudal POm receive substantial inputs from the interpolaris trigeminal subnucleus, whereas the caudal POm also receives additional inputs from the oralis subnucleus (Veinante et al., 2000). Furthermore, whereas caudal POm projects to both DLS and somatosensory (SI) cortex, rostral POm projects to SI cortex, but not to DLS (Ohno et al., 2012).

\section{GABAergic projections from ZIv to $\mathrm{POm}$}

Most neurons in ZIv use GABA as a neurotransmitter (Barthó et al., 2002), and some data indicate that ZIv exerts an inhibitory influence on POm (Trageser and Keller, 2004; Lavallée et al., 2005). To verify that our tracer injections and recording sites in whisker-sensitive parts of POm receive GABAergic projections from ZIv, we placed FG in a whisker-sensitive part of POm and then processed ZI for the presence of GAD, the rate-limiting enzyme for GABA synthesis.

As indicated by confocal images in Figure 8, we observed many FG-labeled neurons in ZIv that were positive for the presence of GAD 65/67. Most of the FG- and GAD-labeled neurons appeared in ZIv, and almost all ZI neurons labeled by FG were also marked by GAD 65/67. Therefore, to the extent that superior colliculus activates ZI neurons that project to $\mathrm{POm}$, collicular activation should inhibit neurons in POm.

\section{Influence of collicular activation on ZI and POm}

To establish that activation of the superior colliculus can excite the incertal pathway to POm, we used optogenetic techniques to evoke neuronal activity in the superior colliculus while recording neuronal responses in ZIv. Because optogenetic methods do not activate fibers of passage, which originate outside the region of ChR2 expression, we could 

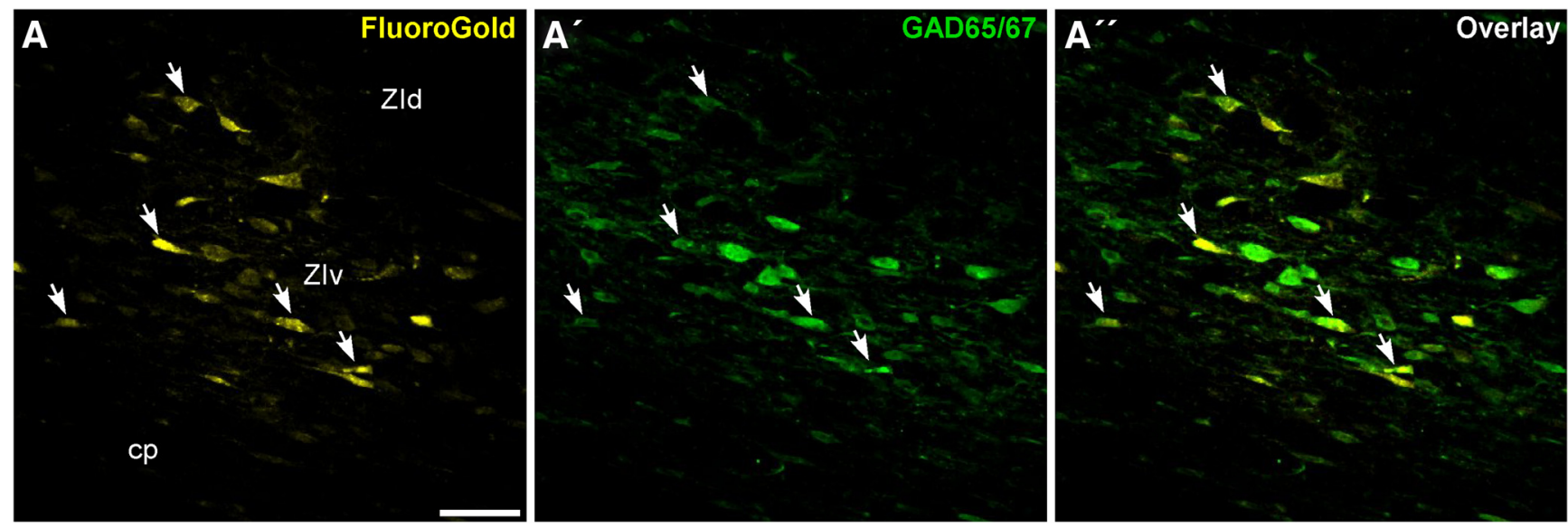

Figure 8. Projections from Zlv to whisker-responsive sites in POm are GABAergic. $A$, Confocal image of retrogradely labeled neurons in Zlv after placing $\mathrm{FG}$ into a whisker-responsive site in POm. $A^{\prime}$, Confocal image of the same stack processed for GABA using an antibody for GAD 65/67. $A^{\prime \prime}, 0$ verlap of FG and GAD fluorescence. White arrows indicate the strongest double-labeled neurons. Scale bar, $50 \mu \mathrm{m}$.
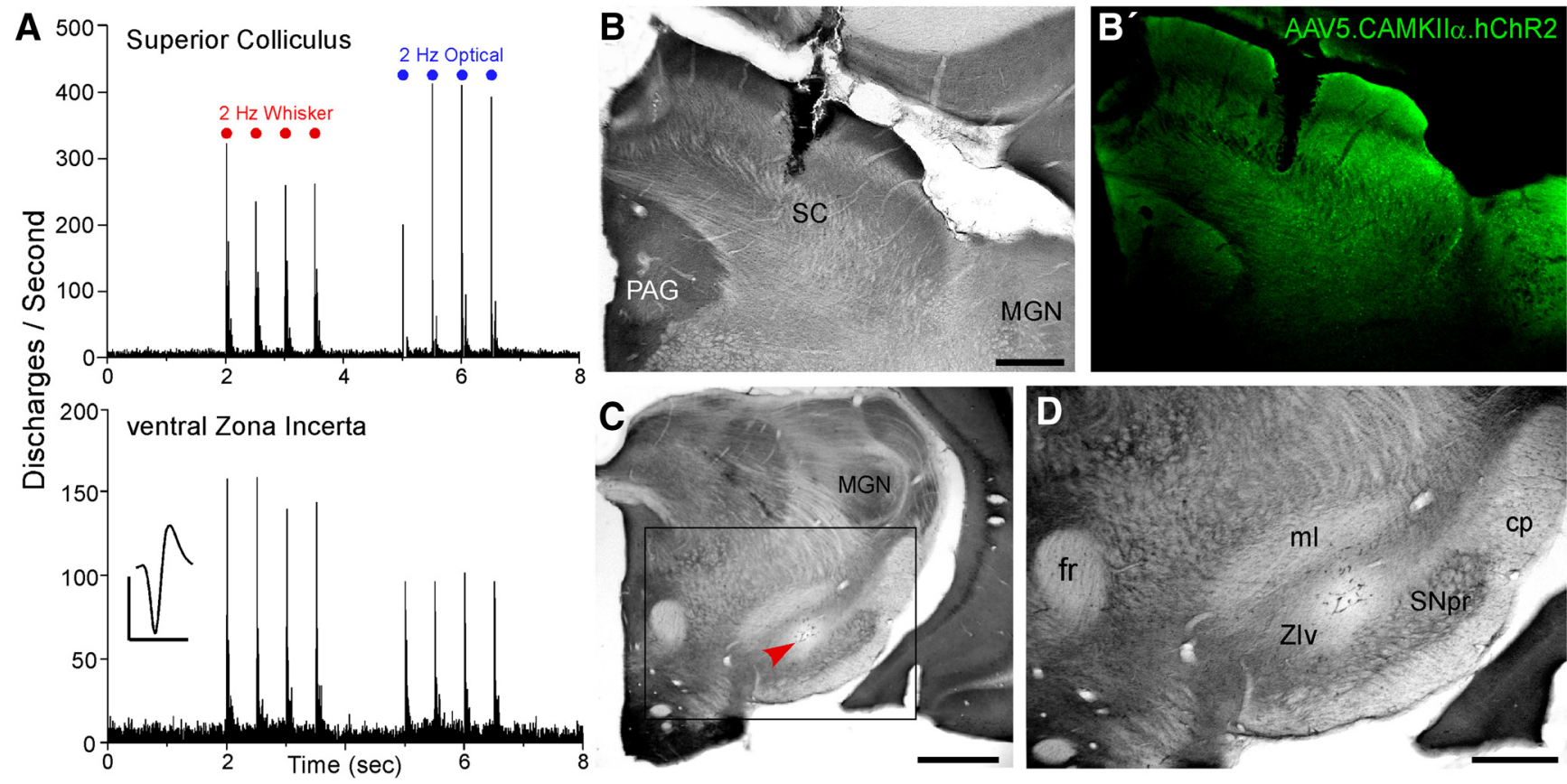

Figure 9. Optical stimulation of superior colliculus evokes neuronal discharges in ZI. A, PSTHs display multiunit responses in the superior colliculus (top) recorded simultaneously with single neuron discharges in Zlv (bottom) during whisker deflections and optical stimulation ( $473 \mathrm{~nm}$ ) of ChR2-expressing neurons in the colliculus. Mean waveform scale: $1 \mathrm{~ms}, 100 \mu \mathrm{V}$. $\boldsymbol{B}, \boldsymbol{B}^{\prime}$, Adjacent sections illustrate the optrode location in the superior colliculus with respect to the laminar organization defined by $C 0$ processing and the expression of ChR2 produced by injections of AAV1.CamKIla.hChR2(H134R)-eYFP.WPRE.hGH (Penn Vector Core). C, D, Low- and high-power views of the neuronal recording site in Zlv (arrow). Inset indicates the region depicted in D.

determine whether selective collicular activation was sufficient to excite neurons in ZIv.

We injected the superior colliculus with an AAV that selectively expresses ChR2 in projection neurons. Figure 9 illustrates an optogenetic experiment in which we recorded neuronal responses to whisker stimulation in both the superior colliculus and ZI before administering a brief train of $50 \mathrm{~ms}$ light pulses to the intermediate layers of the superior colliculus. We tested whisker stimulation to insure that both sites recorded in the superior colliculus and ZIv responded to the same sensory modality, thereby increasing the likelihood that they are interconnected by the colliculo-incertal pathway. In the small sample $(n=7)$ of collicular and incertal neuron pairs that were recorded simultaneously in these experiments, the mean response latency to whis- ker stimulation in the superior colliculus and ZIv was $8.2 \pm 0.5$ and $15.5 \pm 5.5 \mathrm{~ms}$ (mean \pm SEM), respectively.

In two rats we recorded seven neurons in ZIv that responded to optical stimulation of the superior colliculus. Consistent with the example in Figure 9, each ZIv neuron was repetitively excited by the series of light pulses administered to superior colliculus. Across these cases, the latency of ZIv discharges ranged from 2 to $6 \mathrm{~ms}$ after light pulse onset, with a mean latency of $3.0 \pm 0.5 \mathrm{~ms}$. Optogenetic stimulation in superior colliculus was also attempted in one other rat, but it failed to evoke neuronal responses in either the superior colliculus or ZIv of this third case.

In a separate set of rats, the superior colliculus was electrically stimulated while recording spontaneous activity in POm. Biphasic electrical pulses ( $1 \mathrm{~ms}$ duration) ranging from 200 to 

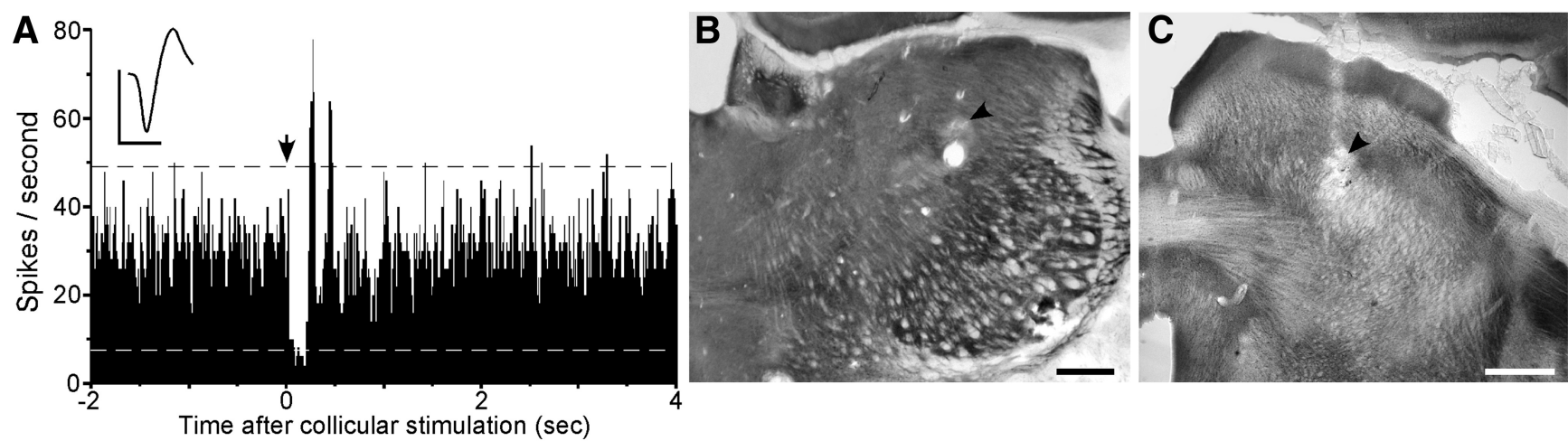

Figure 10. Electrical stimulation in superior colliculus evokes inhibition of spontaneous activity in POm. A, PSTH from a representative POm recording shows the decrease in spontaneous discharges immediately after the superior colliculus is stimulated by a train of five $1 \mathrm{~ms}$ pulses administered at time 0 (arrow). Dashed lines indicate $99 \%$ confidence limits. Mean waveform scales: $1 \mathrm{~ms}, 100 \mu$ V. PSTH: 50 trials, 5 ms binwidths. B, C, Arrows indicate lesions that mark the recording and stimulation sites in P0m and superior colliculus.
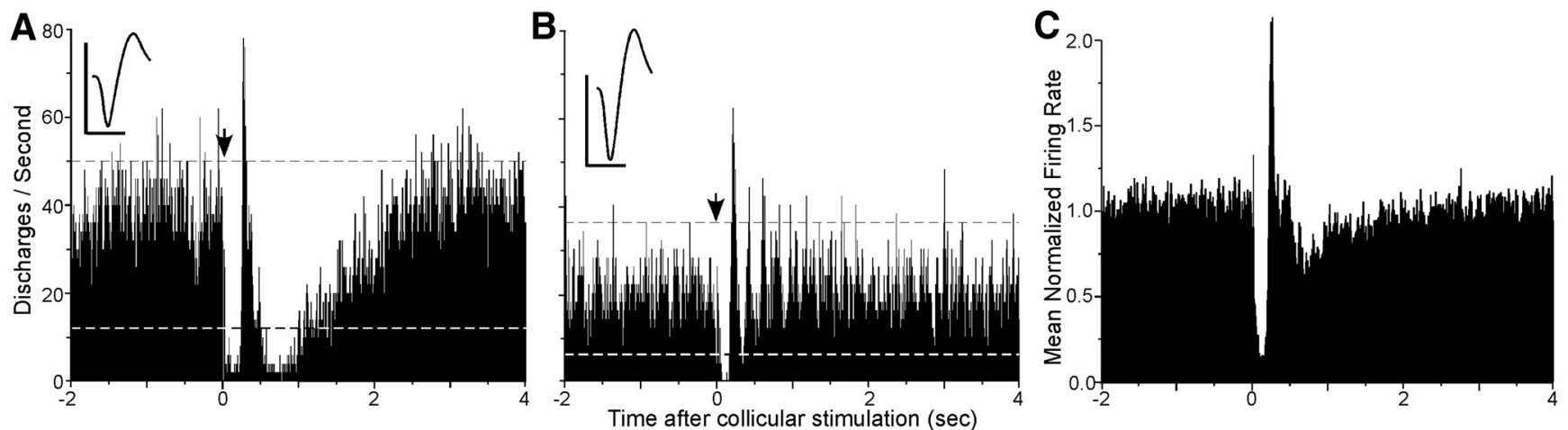

Figure 11. Representative examples and mean responses of POm neurons to electrical stimulation of the superior colliculus. $A, B, P S T H s$ indicate the changes in activity of two neurons in POm that displayed prolonged inhibition $(\boldsymbol{A})$ or oscillations $(\boldsymbol{B})$ in response to electrical stimulation of the superior colliculus (arrows). Dashed lines indicate $99 \%$ confidence limits. Mean waveform scales: $1 \mathrm{~ms}, 100 \mu \mathrm{V}$. C, Mean normalized response of $24 \mathrm{POm}$ neurons. Individual neuronal activity was expressed as a proportion of the mean spontaneous rate before collicular activation, and then the sum of these normalized responses was averaged. Binwidths, $5 \mathrm{~ms}$.

$500 \mu$ A were applied by a concentric bipolar electrode lowered to the intermediate layers of the superior colliculus because our tracing data indicate that these layers are strongly connected with ZIv. We chose electrical stimulation for these experiments to increase the spatial extent of neural activation so that a broader range of collicular projections to ZIv would be excited simultaneously.

Spontaneous POm activity was recorded from nine hemispheres in five lightly anesthetized rats during electrical stimulation of the ipsilateral superior colliculus. All POm neurons were recorded during lightly anesthetized states in which collicular stimulation evoked protracted movements of the ipsilateral whiskers. Before collicular stimulation, we established that each POm neuron either responded to whisker deflections or was in close proximity $(\sim 100-200 \mu \mathrm{m})$ to other POm neurons that responded to whisker stimulation.

After initial experiments testing one or two pulses of biphasic stimulation to activate the superior colliculus, we found that a train of five $1 \mathrm{~ms}$ pulses administered over a $10 \mathrm{~ms}$ interval would reliably evoke significant decreases in POm spontaneous activity (see Materials and Methods). From a sample of 27 neurons recorded in POm, electrical stimulation of the superior colliculus evoked a significant decrease in the spontaneous activity in 24 of them. In these 24 cases, spontaneous activity declined below the $99 \%$ confidence limits and was followed by a significant increase in discharge rate that also exceeded the $99 \%$ confidence limits.
An example of collicular-induced inhibition of POm activity is illustrated in Figure 10. As indicated by the PSTH, collicular stimulation evoked a rapid and significant decrease in spontaneous activity that persisted for $185 \mathrm{~ms}$. Following this inhibitory period, the POm neuron displayed a rebound in the rate of neuronal discharges that exceeded the $99 \%$ confidence limits. In addition, a second period of increased activity followed the first peak $\sim 150$ ms later.

Electrical stimulation of the superior colliculus produced a reliable pattern of inhibition followed by excitation in $\mathrm{POm}$, but subsequent changes in neuronal activity varied considerably across the sample of recorded neurons. In some cases, illustrated in Figure $11 \mathrm{~A}$, the sequential phase of inhibition and excitation was followed by a prolonged inhibitory period $(\sim 2 \mathrm{~s})$ in which the rate of neuronal discharges gradually returned to the baseline rate. In other cases (Fig. 11B), the initial sequence of inhibition and excitation was followed by a short series of oscillations. In some of these instances, the sequence of valleys and peaks exceeded the confidence limits, but in other cases, only the excitatory peaks exceeded the confidence limits.

As shown by Figure $11 C$, the mean population response to collicular stimulation was dominated by an initial period of inhibition followed by a rebound of spontaneous activity. To obtain this population response, we normalized the responses of all POm neurons by dividing each bin throughout each individual PSTH by the mean rate of activity in the $2 \mathrm{~s}$ period 


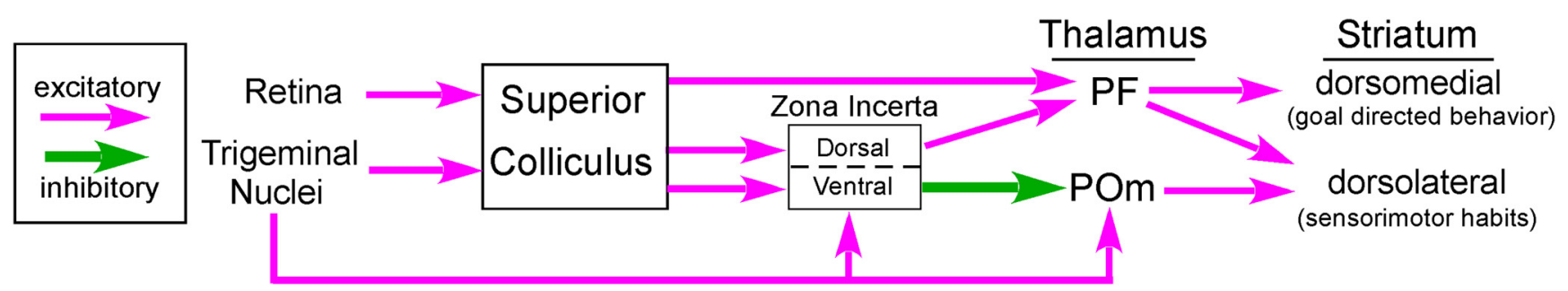

Figure 12. Circuit connections by which the superior colliculus exerts opposing influences on Pf and POm. Superior colliculus activates Pf while simultaneously activating inhibitory projections from Zl to POm. Incertal-mediated inhibition of the POm projections to the dorsolateral striatum could disrupt well learned behavioral sequences that comprise sensorimotor habits.

before electrical stimulation. Electrical stimulation artifacts prevented an accurate determination of the latency of inhibition in POm, but it was apparent that spontaneous activity had declined significantly by the time that collicular stimulation had ceased. On average, the subsequent rebound in spontaneous activity reached its peak $130 \mathrm{~ms}$ after electrical stimulation had stopped. Because of variations in the subsequent pattern of activity across our neuronal sample, close examination of the population PSTH after the first peak reveals small oscillations in activity that are superimposed on a longer period of inhibition.

\section{Discussion}

This study demonstrates that superior colliculus projects to ZIv regions that innervate POm. Furthermore, we found that direct stimulation of superior colliculus causes activation of ZIv and inhibition of spontaneous activity in POm. By regulating communication between the superior colliculus and $\mathrm{POm}$, the colliculo-incertal-POm pathway provides a circuit mechanism by which salient, unexpected sensory events can inhibit POm-DLS interactions, thereby disrupting DLS processing and the execution of ongoing behavioral routines. The inhibitory projections from ZIv to POm may also account for clinical data indicating that deep brain stimulation of $\mathrm{ZI}$ is beneficial for treating essential tremor and symptoms associated with PD (Plaha et al., 2008, 2011; Blomstedt et al., 2012; Fytagoridis et al., 2012; Caire et al., 2013).

\section{Comparison of collicular and pallidal connections}

Some recent studies had reported that the superior colliculus projects strongly to STN (Coizet et al., 2009; Redgrave et al., 2010a). Therefore, we injected an anterograde tracer into superior colliculus and a retrograde tracer into globus pallidus to assess the labeling patterns in STN and surrounding regions. Contrary to the view that superior colliculus projects to STN, we observed two separate areas of dense labeling: (1) anterogradely labeled projection terminals in ZI, and (2) retrogradely labeled neurons in STN. These results support previous studies that had emphasized STN connections with the globus pallidus but did not observe any STN connections with the superior colliculus (Ricardo, 1980; Canteras et al., 1990).

We examined the labeled collicular projections with respect to STN boundaries defined by Nissl staining and the presence of cytochrome oxidase. In the present study and in previous work (Alloway et al., 2014), we found that placing anterograde tracers in the superior colliculus consistently produced dense arbors of labeled terminals in ZI. Sparse terminal labeling was observed along the dorsal edge of the STN, but it represented only $2 \%$ of total labeling in ZI. Indeed, labeled projections from the superior colliculus innervated extensive parts of ZI, including regions lo- cated rostral, caudal, medial, and lateral to the boundaries of STN.

\section{Collicular projections to the zona incerta}

Our data confirm older studies that identified a pathway from the superior colliculus to ZIv (Roger and Cadusseau, 1985; ShammahLagnado et al., 1985; Kolmac et al., 1998). Furthermore, we observed a crude topographical organization in which the medial part of the superior colliculus, which responds preferentially to visual stimulation, projects to the most lateral part of ZIv. The lateral superior colliculus, by comparison, responds best to whisker deflections and projects to more medial parts of ZIv.

This topography is consistent with anatomical evidence suggesting that visual and somesthetic information is transmitted to the lateral and medial parts of ZI, respectively ( $\mathrm{Ni}$ colelis et al., 1992; Power et al., 2001, Shaw and Mitrofanis, 2002). Although one study reported that collicular projections to ZI do not possess any topographic organization (Kolmac et al., 1998), that report did not depict any tracer deposits in the medial superior colliculus. The same study, however, reported that lateral ZI projects to the medial superior colliculus, whereas medial ZI projects to the lateral superior colliculus. By injecting both retrograde and anterograde tracers in widely separated regions that were physiologically defined, our work indicates a crossed pattern of reciprocal connections between ZI and the superior colliculus.

\section{Incertal control of thalamostriatal projections from POm}

Our recordings indicate that the extreme lateral ZI responds to visual stimulation while more medial regions respond to whisker defections. Despite these functional differences, focal deposits of $\mathrm{BDA} / \mathrm{FG}$ into light-sensitive or whisker-sensitive parts of ZIv revealed extensive populations of retrogradely labeled neurons in the superior colliculus that were coupled with anterogradely labeled terminals in POm. When retrograde tracer injections in whisker-sensitive parts of caudal POm were paired with anterograde tracer deposits in superior colliculus, we consistently observed tracer overlap in ZIv regardless of whether we injected whisker- or retina-sensitive parts of the superior colliculus. We also found that most FG-labeled neurons in ZIv were associated with GAD 65/67, which is a GABAergic marker.

Consistent with these findings, direct stimulation of the superior colliculus caused activation of ZIv and inhibition of spontaneous discharges in caudal POm. Although caudal POm has not been thoroughly described, most neurons in that region are presumed to be thalamocortical projection neurons that send collateral projections to the DLS (Deschênes et al., 1995; Ohno et al., 2012). If this view is correct, collicularevoked inhibition of caudal POm should inhibit its projections to DLS. 
These findings are significant because the superior colliculus coordinates the neural circuits that redirect attention and alter behavioral responses to unexpected sensory events (Stein et al., 2004). Selective optogenetic stimulation of corticotectal projections from visual cortex causes an immediate arrest of behavior (Liang et al., 2015), and this is consistent with collicular projections to ZIv. Hence, sensory events that activate the superior colliculus could inhibit POm neurons that project to DLS. The DLS is essential for executing well learned behavioral routines in a familiar context (Yin et al., 2004, 2006; Balleine and O'Doherty, 2010), and this function appears to depend on S-R associations mediated by sensory inputs from POm and related parts of sensorimotor cortex (Mowery et al., 2011). Therefore, as part of the circuitry for executing responses to unexpected sensory events, the colliculo-incertalPOm pathway provides a mechanism for inhibiting POmDLS interactions that facilitate specific $\mathrm{S}-\mathrm{R}$ behavioral sequences.

\section{Circuits for activating incertal inhibition}

We found that in the lightly anesthetized state, whisker deflections could evoke neuronal responses in the superior colliculus, ZIv, and POm. However, if the superior colliculus activates ZIv, then it seems reasonable to predict that POm neurons should be inhibited by ZIv output. However, this logic neglects consideration of likely differences in the complex circuit dynamics that transpire in anesthetized and awake, behaving animals. During conscious behavior, for example, projections from sensorimotor cortex to POm and ZI are likely to play an important role in determining how ZI regulates POm activity (Urbain and Deschênes, 2007).

In addition to the superior colliculus, ZIv also receives ascending sensory inputs from the trigeminal nuclei (Williams et al., 1994). How colliculo-incertal and trigemino-incertal projections act together to influence the inhibitory output from ZIv is complicated by the presence of intrinsic interconnections within ZIv that could disinhibit the GABAergic projections to POm in some circumstances (Power and Mitrofanis, 1999; Urbain and Deschênes, 2007).

Our small sample of simultaneous neuronal recordings in ZIv and superior colliculus during whisker deflections indicated that superior colliculus was activated, on average, $\sim 7 \mathrm{~ms}$ before neurons in ZIv. We also found that the optogenetic delay for transmitting information from superior colliculus to $\mathrm{ZIv}$ is $\sim 3.0 \mathrm{~ms}$. This suggests that an unexpected whisker deflection could excite superior colliculus and that the output of the colliculo-incertal pathway could act synergistically with the trigeminal projections to ZIv.

Although the precise mechanisms that control incertal inhibition of POm during different behavioral states and environmental contexts have not been fully elucidated, our results demonstrate that collicular activation can inhibit POm activity. This is significant because the superior colliculus is important for coordinating the neural circuits that enable the behavioral changes and redirection of attention that occur in response to unexpected sensory events (Stein et al., 2004).

\section{Thalamostriatal circuit mechanisms}

As portrayed in Figure 12, there are parallel projections from the superior colliculus that could work together to halt an ongoing behavior so that more appropriate responses are selected. In addition to the colliculo-incertal-POm pathway, the superior colliculus projects to the Pf thalamic nucleus (Krout et al., 2001; Alloway et al.,
2014). In brain slices, stimulation of the intralaminar thalamic nuclei activate cholinergic interneurons in the dorsal striatum, which leads to a burst-pause response and subsequent increases in the output of the indirect striatal-pallidal pathway (Ding et al., 2010). The Pf and related nuclei respond to unexpected sensory events (Matsumoto et al., 2001; Minamimoto and Kimura, 2002), and these findings have prompted the view that activation of the intralaminar thalamic nuclei pauses striatal processing so that a new behavior can be selected (Thorn and Graybiel, 2010). Hence, collicular activation of Pf and its projections to the striatum could complement inhibition exerted simultaneously by ZI on caudal POm and its interactions with the DLS.

\section{Clinical significance of the zona incerta}

The STN is an important target for deep brain stimulation in the treatment of PD. Given the proximity of STN and ZI, it is not surprising that CT scans, structural MRIs, and postmortem analysis indicate that DBS electrode contacts are often located in ZI (Sun et al., 2008; Plaha et al., 2011; Blomstedt et al., 2012; Fytagoridis et al., 2012; Caire et al., 2013). In these cases, electrical stimulation of the caudal sector of ZI, which sends excitatory projections to the other sectors of ZI (Power and Mitrofanis, 1999; Mitrofanis, 2005), is effective for treating essential tremor, as well as some motor symptoms associated with PD. In view of the data in the present study, it is plausible that the therapeutic benefits derived from ZI stimulation are due, at least in part, to its disruptive effects on the inhibitory outputs of ZIv, which should inhibit POm-DLS interactions.

\section{References}

Aligny C, Roux C, Dourmap N, Ramdani Y, Do-Rego JC, Jégou S, Leroux P, Leroux-Nicollet I, Marret S, Gonzalez BJ (2014) Ketamine alters cortical integration of GABAergic interneurons and induces long-term sexdependent impairments in transgenic Gad67-GFP mice. Cell Death Dis 5:e1311. CrossRef Medline

Alloway KD, Hoffer ZS, Hoover JE (2003) Quantitative comparisons of corticothalamic topography within the ventrobasal complex and the posterior nucleus of the rodent thalamus. Brain Res 968:54-68. CrossRef Medline

Alloway KD, Smith JB, Watson GD (2014) Thalamostriatal projections from the medial posterior and parafascicular nuclei have distinct topographic and physiologic properties. J Neurophysiol 111:36-50. CrossRef Medline

Balleine BW, O’Doherty JP (2010) Human and rodent homologies in action control: corticostriatal determinants of goal-directed and habitual action. Neuropsychopharmacology 35:48-69. CrossRef Medline

Barthó P, Freund TF, Acsády L (2002) Selective GABAergic innervation of thalamic nuclei from zona incerta. Eur J Neurosci 16:999-1014. CrossRef Medline

Blomstedt P, Fytagoridis A, Åström M, Linder J, Forsgren L, Hariz MI (2012) Unilateral caudal zona incerta deep brain stimulation for Parkinsonian tremor. Parkinsonism Relat Disord 18:1062-1066. CrossRef Medline

Caire F, Ranoux D, Guehl D, Burbaud, Cuny E (2013) A systematic review of studies on anatomical position of electrode contacts for chronic subthalamic stimulation in Parkinson's disease. Acta Neurochir 155:16471654. CrossRef Medline

Canteras NS, Shammah-Lagnado SJ, Silva BA, Ricardo JA (1990) Afferent connections of the subthalamic nucleus: a combined retrograde and anterograde horseradish peroxidase study in the rat. Brain Res 513:43-59. CrossRef Medline

Cohen JD, Hirata A, Castro-Alamancos MA (2008) Vibrissa sensation in superior colliculus: wide-field sensitivity and state-dependent cortical feedback. J Neurosci 28:11205-11220. CrossRef Medline

Coizet V, Graham JH, Moss J, Bolam JP, Savasta M, McHaffie JG, Redgrave P, Overton PG (2009) Short-latency visual input to the subthalamic nucleus is provided by the midbrain superior colliculus. J Neurosci 29:57015709. CrossRef Medline 
Comoli E, Coizet V, Boyes J, Bolam JP, Canteras NS, Quirk RH, Overton PG, Redgrave P (2003) A direct projection from superior colliculus to the substantia nigra for detecting salient visual events. Nat Neurosci 6:974980. CrossRef Medline

Deschênes M, Bourassa J, Parent A (1995) Two different types of thalamic fibers innervate the rat striatum. Brain Res 701:288-292. CrossRef Medline

Diamond ME, Armstrong-James M, Budway MJ, Ebner FF (1992) Somatic sensory responses in the rostral sector of the posterior group (POm) and in the ventral posterior medial nucleus (VPM) of the rat thalamus: dependence on the barrel field cortex. J Comp Neurol 319:66-84. CrossRef Medline

Ding JB, Guzman JN, Peterson JD, Goldberg JA, Surmeier DJ (2010) Thalamic gating of corticostriatal signaling by cholinergic interneurons. Neuron 67:294-307. CrossRef Medline

Friedberg MH, Lee SM, Ebner FF (1999) Modulation of receptive field properties of thalamic somatosensory neurons by the depth of anesthesia. J Neurophysiol 81:2243-2252. Medline

Fytagoridis A, Sandvik U, Aström M, Bergenheim T, Blomstedt P (2012) Long-term follow-up of deep brain stimulation of the caudal zona incerta for essential tremor. J Neurol Neurosurg Psychiatry 83:258-262. CrossRef Medline

Graybiel AM (2008) Habits, rituals, and the evaluative brain. Annu Rev Neurosci 31:359-387. CrossRef Medline

Groenwegen HJ, Berendse HW (1990) Connections of the subthalamic nucleus with ventral striatopallidal parts of the basal ganglia in the rat. J Comp Neurol 294:607-622. CrossRef Medline

Groh A, Bokor H, Mease RA, Plattner VM, Hangya B, Stroh A, Deschenes M, Acsády L (2014) Convergence of cortical and sensory driver inputs on single thalamocortical cells. Cereb Cortex 24:3167-3179. CrossRef Medline

Grunwerg BS, Krauthamer GM (1990) Vibrissa-responsive neurons of the superior colliculus that project to the intralaminar thalamus of the rat. Neurosci Lett 111:23-37. CrossRef Medline

Grunwerg BS, Krauthamer GM (1992) Sensory responses of intralaminar thalamic neurons activated by the superior colliculus. Exp Brain Res 88: 541-550. Medline

Kincaid AE, Wilson CJ (1996) Corticostriatal innervation of the patch and matrix in the rat striatum. J Comp Neurol 374:578-592. CrossRef Medline

Kita H, Kitai ST (1987) Efferent projections of the subthalamic nucleus in the rat: light and electron microscopic analysis with the PHA-L method. J Comp Neurol 260:435-452. CrossRef Medline

Kolmac CI, Power BD, Mitrofanis J (1998) Patterns of connections between zona incerta and brainstem in rats. J Comp Neurol 396:544-555. CrossRef Medline

Krout KE, Loewy AD, Westby GW, Redgrave P (2001) Superior colliculus projections to midline and intralaminar thalamic nuclei of the rat. J Comp Neurol 431:198-216. CrossRef Medline

Land PW, Simons DJ (1985) Cytochrome oxidase staining in the rat SmI barrel cortex. J Comp Neurol 238:225-235. CrossRef Medline

Lavallée P, Urbain N, Dufresne C, Bokor H, Acsády L, Deschênes M (2005) Feedforward inhibitory control of sensory information in higher-order thalamic nuclei. J Neurosci 25:7489-7498. CrossRef Medline

Liang F, Xiong XR, Zingg B, Ji XY, Zhang LI, Tao HW (2015) Sensory cortical control of a visually induced arrest behavior via corticotectal projections. Neuron 86:755-767. CrossRef Medline

Matsumoto N, Minamimoto T, Graybiel AM, Kimura M (2001) Neurons in the thalamic CM-Pf complex supply striatal neurons with information about behaviorally significant sensory events. J Neurophysiol 85:960976. Medline

Meng Z, Li Q, Martin JH (2004) The transition from development to motor control function in the corticospinal system. J Neurosci 24:605-614. CrossRef Medline

Minamimoto T, Kimura M (2002) Participation of the thalamic CM-Pf complex in attentional orienting. J Neurophysiol 87:3090-3101. Medline

Mitrofanis J (2005) Some certainty for the "zone of uncertainty"? Exploring the function of the zona incerta. Neuroscience 130:1-15. CrossRef Medline

Mowery TM, Harrold JB, Alloway KD (2011) Repeated whisker stimulation evokes invariant neuronal responses in the dorsolateral striatum of anesthetized rats: a potential correlate of sensorimotor habits. J Neurophysiol 105:2225-2238. CrossRef Medline
Nicolelis MA, Chapin JK, Lin RC (1992) Somatotopic maps within zona incerta relay parallel GABAergic somatosensory pathways to the neocortex, superior colliculus, and brainstem. Brain Res 577:134-141. CrossRef Medline

Ohno S, Kuramoto E, Furuta T, Hioki H, Tanaka YR, Fujiyama F, Sonomura T, Uemura M, Sugiyama K, Kaneko T (2012) A morphological analysis of thalamocortical axon fibers of rat posterior thalamic nuclei: a single neuron tracing study with viral vectors. Cereb Cortex 22:2840-2857. CrossRef Medline

Oostland M, Sellmeijer J, van Hooft JA (2011) Transient expression of functional serotonin 5-HT3 receptors by glutamatergic granule cells in the early postnatal mouse cerebellum. J Physiol 589:4837-4846. CrossRef Medline

Paxinos G, Watson C (2005) The rat brain in stereotaxic coordinates, Ed 6. New York: Academic.

Plaha P, Khan S, Gill SS (2008) Bilateral stimulation of the caudal zona incerta nucleus for tremor control. J Neurol Neurosurg Psychiatry 79: 504-513. CrossRef Medline

Plaha P, Javed S, Agombar D, O’Farrell G, Khan S, Whone A, Gill S (2011) Bilateral caudal zona incerta nucleus stimulation for essential tremor: outcome and quality of life. J Neurol Neurosurg Psychiatry 82:899-904. CrossRef Medline

Power BD, Mitrofanis J (1999) Evidence for extensive inter-connections within the zona incerta in rats. Neurosci Lett 267:9-12. CrossRef Medline

Power BD, Leamey CA, Mitrofanis J (2001) Evidence for a visual subsector within zona incerta. Vis Neurosci 18:179-186. CrossRef Medline

Redgrave P, Coizet V, Comoli E, McHaffie JG, Leriche M, Vautrelle N, Hayes LM, Overton P (2010a) Interactions between the midbrain superior colliculus and the basal ganglia. Front Neuroanat 4:132. CrossRef Medline

Redgrave P, Rodriguez M, Smith Y, Rodriguez-Oroz MC, Lehericy S, Bergman H, Agid Y, DeLong MR, Obeso JA (2010b) Goal-directed and habitual control in the basal ganglia: implications for Parkinson's disease. Nat Rev Neurosci 11:760-772. CrossRef Medline

Ricardo JA (1980) Efferent connections of the subthalamic region in the rat: I. The subthalamic nucleus of Luys. Brain Res 202:257-271. CrossRef Medline

Roger M, Cadusseau J (1985) Afferents to the zona incerta in the rat: a combined retrograde and anterograde study. J Comp Neurol 241:480492. CrossRef Medline

Shammah-Lagnado SJ, Negrão N, Ricardo JA (1985) Afferent connections of the zona incerta: a horseradish peroxidase study in the rat. Neuroscience 15:109-134. CrossRef Medline

Shaw V, Mitrofanis J (2002) Anatomical evidence for somatotopic maps in the zona incerta of rats. Anat Embryol 206:119-130. CrossRef Medline

Smith JB, Alloway KD (2010) Functional specificity of claustrum connections in the rat: interhemispheric communication between specific parts of motor cortex. J Neurosci 30:16832-16844. CrossRef Medline

Smith JB, Alloway KD (2013) Rat whisker motor cortex is subdivided into sensory-input and motor-output areas. Front Neural Circuits 7:4. CrossRef Medline

Smith JB, Alloway KD (2014) Interhemispheric claustrum circuits coordinate somatomotor and visuomotor networks that regulate bilateral behaviors. Front Syst Neurosci 8:93. CrossRef Medline

Smith JB, Mowery TM, Alloway KD (2012) Thalamic POm projections to the dorsolateral striatum of rats: potential pathway for mediating stimulus-response associations for sensorimotor habits. J Neurophysiol 108:160-174. CrossRef Medline

Sparks DL (1986) Translation of sensory signals into commands for control of saccadic eye movements: role of the primate superior colliculus. Physiol Rev 66:118-171. Medline

Stein BE, Jiang W, Stanford TR (2004) Multisensory integration in single neurons of the midbrain. In: Handbook of multisensory integration (Calvert G, Stein BE, eds), pp 243-264. Cambridge MA: MIT.

Sun DA, Yu H, Spooner J, Tatsas AD, Davis T, Abel TW, Kao C, Konrad PE (2008) Postmortem analysis following 71 months of deep brain stimulation of the subthalamic nucleus for Parkinson's disease. J Neurosurg 109: 325-329. CrossRef Medline

Thorn CA, Graybiel AM (2010) Pausing to regroup: thalamic gating of cortico-basal ganglia networks. Neuron 67:175-178. CrossRef Medline

Tokuno H, Takada M, Ikai Y, Mizuno N (1994) Direct projections from the deep layers of the superior colliculus to the subthalamic nucleus in the rat. Brain Res 639:156-160. CrossRef Medline 
Trageser JC, Keller A (2004) Reducing the uncertainty: gating of peripheral inputs by zona incerta. J Neurosci 24:8911-8915. CrossRef Medline

Urbain N, Deschênes M (2007) Motor cortex gates vibrissal responses in a thalamocortical projection pathway. Neuron 56:714-725. CrossRef Medline

Veinante P, Jacquin MF, Deschênes M (2000) Thalamic projections from the whisker-sensitive regions of the spinal trigeminal complex in the rat. J Comp Neurol 420:233-243. CrossRef Medline

Voigt T, De Lima AD, Beckmann M (1993) Synatophysin immunohistochemistry reveals inside-out pattern of early synaptogenesis in ferret cerebral cortex. J Comp Neurol 330:48-64. CrossRef Medline

Watson C, Lind CR, Thomas MG (2014) The anatomy of the caudal zona incerta in rodents and primates. J Anat 224:95-107. CrossRef Medline
Williams MN, Zahm DS, Jacquin MF (1994) Differential foci and synaptic organization of the principal and spinal trigeminal projections to the thalamus in the rat. Eur J Neurosci 6:429-453. CrossRef Medline

Yin HH, Knowlton BJ, Balleine BW (2004) Lesions of the dorsolateral striatum preserve outcome expectancy but disrupt habit formation in instrumental learning. Eur J Neurosci 19:181-189. CrossRef Medline

Yin HH, Knowlton BJ, Balleine BW (2006) Inactivation of dorsolateral striatum enhances sensitivity to changes in the action-outcome contingency in instrumental conditioning. Behav Brain Res 166:189-196. CrossRef Medline

Zhang M, Alloway KD (2004) Stimulus-induced intercolumnar synchronization of neuronal activity in rat barrel cortex: a laminar analysis. J Neurophysiol 92:1464-1478. CrossRef Medline 